\title{
Operator evolution via the similarity renormalization group: The deuteron
}

\author{
E. R. Anderson, ${ }^{1, *}$ S. K. Bogner ${ }^{2, \dagger}$ R. J. Furnstahl, ${ }^{1, \dagger}$ and R. J. Perry ${ }^{1, \S}$ \\ ${ }^{1}$ Department of Physics, The Ohio State University, Columbus, Ohio 43210, USA \\ ${ }^{2}$ National Superconducting Cyclotron Laboratory and Department of Physics and Astronomy, \\ Michigan State University, East Lansing, Michigan 48824, USA \\ (Received 30 August 2010; published 3 November 2010)
}

\begin{abstract}
Similarity renormalization group (SRG) flow equations can be used to unitarily soften nuclear Hamiltonians by decoupling high-energy intermediate-state contributions to low-energy observables while maintaining the natural hierarchy of many-body forces. Analogous flow equations can be used to consistently evolve operators so that observables are unchanged if no approximations are made. The question in practice is whether the advantages of a softer Hamiltonian and less-correlated wave functions might be offset by complications in approximating and applying other operators. Here we examine the properties of SRG-evolved operators, focusing in this article on applications to the deuteron but leading toward methods for few-body systems. We find the advantageous features generally carry over to other operators with additional simplifications in some cases from factorization of the unitary transformation operator.
\end{abstract}

DOI: 10.1103/PhysRevC.82.054001

PACS number(s): 21.30.-x, 05.10.Cc, 13.75.Cs

\section{INTRODUCTION}

Renormalization group methods can be used to soften interactions in nuclear systems, which extends the range of many computational methods and qualitatively improves their convergence patterns [1]. The similarity renormalization group (SRG) [2-6] does this by systematically evolving Hamiltonians via a continuous series of unitary transformations chosen to decouple the high- and low-energy matrix elements of a given interaction $[7,8]$. In particular, a flow equation with parameter $s$ and generator $\eta_{s} \equiv\left[G_{s}, H_{s}\right]$,

$$
\frac{d H_{s}}{d s}=\left[\eta_{s}, H_{s}\right]
$$

unitarily evolves an initial Hamiltonian $H_{s=0} \equiv H=T_{\text {rel }}+V$. Choosing the flow operator $G_{s}$ specifies the SRG evolution. This equation implements the unitary transformation

$$
H_{s}=U_{s} H_{s=0} U_{s}^{\dagger}=T_{\text {rel }}+V_{s},
$$

which defines $V_{s}$ by choosing the relative kinetic energy to be invariant and where the generator $\eta_{s}$ is related to $U_{s}$ by

$$
\eta_{s}=\frac{d U_{s}}{d s} U_{s}^{\dagger}=-\eta_{s}^{\dagger} .
$$

As in most previous nuclear applications, here we take $G_{s}=$ $T_{\text {rel }}$ to suppress off-diagonal elements of the Hamiltonian in momentum space (see Sec. II; alternative choices are discussed in Refs. [9] and [10]). This decoupling leads to greatly improved convergence of the binding energy in fewand many-body calculations [8,11]. A major advantage of the SRG relative to other energy-independent renormalization group ( $R G$ ) methods is that the Hamiltonian flow equation is formulated solely in terms of the evolving Hamiltonian

\footnotetext{
*anderson@physics.ohio-state.edu

†bogner@nscl.msu.edu

†furnstahl.1@osu.edu

§perry@mps.ohio-state.edu
}

and does not involve the $T$-matrix, which avoids issues with solving equations in multiple channels and allows any convenient basis to be used; these features mean that evolving few-body forces is practical $[8,12]$.

To use the wave functions produced by SRG-evolved interactions to calculate other matrix elements of interest, we cannot in general neglect the associated change in operators. The evolution of any operator $\widehat{O} \equiv \widehat{O}_{s=0}$ is given by the same unitary transformation used to evolve the Hamiltonian $[4,13]$,

$$
\widehat{O}_{s}=U_{s} \widehat{O}_{s=0} U_{s}^{\dagger},
$$

which implies by differentiation with respect to $s$ the general operator SRG equation,

$$
\frac{d \widehat{O}_{s}}{d s}=\left[\eta_{s}, \widehat{O}_{s}\right] .
$$

Although this equation can be used to find $\widehat{O}_{s}$, it is computationally efficient to construct the unitary transformation directly from the eigenvectors of the evolved and unevolved Hamiltonian using

$$
U_{s}=\sum_{\alpha}\left|\psi_{\alpha}(s)\right\rangle\left\langle\psi_{\alpha}(0)\right|,
$$

where the sum on $\alpha$ is over all eigenvectors, and then to apply Eq. (4) directly. In practice, we work in a discretized basis, so this sum is finite and Eq. (4) is a simple matrix product. In cases where both methods have been used to calculate the SRG evolution, the transformations produced by Eqs. (5) and (6) agree up to numerical errors. However, it has been found that the one-step transformation produced from the eigenvectors is numerically more robust than the differential equation. The direct construction of $U_{s}$ via Eq. (6) is used to calculate operator evolution throughout this work.

If implemented without approximation, unitary transformations preserve operator matrix elements by construction,

$$
\left\langle\psi_{\alpha}(s)\left|\widehat{O}_{s}\right| \psi_{\alpha^{\prime}}(s)\right\rangle=\left\langle\psi_{\alpha}(0)\left|\widehat{O}_{s=0}\right| \psi_{\alpha^{\prime}}(0)\right\rangle,
$$


and thus preserve the physics in the initial Hamiltonian and other operators. But do the advantages of the SRG evolution of Hamiltonians carry over to other operators and are there problems with the practical implementation of operator evolution? Equation (7) implies that changes in the wave function are "compensated" by changes in the operator. This might reasonably be expected to shift around the physics while conserving the computational difficulty, so that at best we must deal with either a simple operator and complicated wave functions or a complicated operator and simplified wave functions. However, the SRG evolution of the Hamiltonian generates a much simpler interaction (smoother and decoupled), which leads to a simpler wave function (reduced shortrange correlations). ${ }^{1}$ What about other operators? Could there be strong and/or fine-tuned cancellations between the evolved wave functions and evolved operators? Can the hierarchy of many-body contributions be violated? We address these questions in this article and a sequel [10].

The evolution of three-body and higher-body interactions is critically important for the SRG and a parallel discussion is needed for other operators. To see how one-, two-, three-, and higher-body operators can be identified, it is useful to decompose the running SRG operator $\widehat{O}_{s}$ in second-quantized form. Schematically (suppressing indices and sums),

$$
\widehat{O}_{s}=\left\langle\widehat{O}_{s}^{(1)}\right\rangle a^{\dagger} a+\left\langle\widehat{O}_{s}^{(2)}\right\rangle a^{\dagger} a^{\dagger} a a+\left\langle\widehat{O}_{s}^{(3)}\right\rangle a^{\dagger} a^{\dagger} a^{\dagger} a a a+\cdots
$$

where $a^{\dagger}$ and $a$ are creation and annihilation operators with respect to the vacuum in a single-particle basis. This defines $\left\langle\widehat{O}_{s}^{(1)}\right\rangle,\left\langle\widehat{O}_{s}^{(2)}\right\rangle,\left\langle\widehat{O}_{s}^{(3)}\right\rangle, \ldots$ as the one-body, two-body, threebody, ... operator matrix elements in that basis at each $s$. The SRG evolution in Eqs. (1) and (5) is dictated by commutators involving $\widehat{O}_{s}$ and $H_{s}$ (which also has such an expansion). When they are evaluated, we see that even if initially there are only one-body operators, higher-body terms will appear in both $\widehat{O}_{S}$ and $H_{s}$ with each step in $s$. Thus, when applied in an $A$-body subspace, the SRG will "induce" $A$-body operators. However, we find that each $\left\langle\widehat{O}_{s}^{(n)}\right\rangle$ is determined fully in the $A=n$ subspace, with no dependence on higher-body operators. This allows us to extract the induced many-body components of the operator as needed [10]. Note that for Hamiltonians with no external potentials (i.e., no one-body interactions), $\left\langle\widehat{O}_{s}^{(1)}\right\rangle$ is independent of $s[10]$. It is also important to remember that input operators, $\widehat{O}_{s=0}$, for low-energy effective theories are generally never simply one- or two-body operators, although these components may dominate. The question is whether an initial many-body hierarchy (expected from chiral effective field theory formulations of the nuclear interaction) is maintained by the SRG evolution.

To avoid confusion, we note that it is also possible to normal order with respect to a finite-density reference state instead of the vacuum, which leads to the "in-medium SRG" (see Ref. [14] and references therein). This changes the definition of the matrix elements and creation and annihilation operators

\footnotetext{
${ }^{1}$ Note that the interpretation of "simpler" can vary. For some Monte Carlo methods, the SRG Hamiltonians become more complicated in the sense of increasing nonlocality in coordinate representation.
}

in Eq. (8) and shifts higher-body pieces to the zero-body, onebody, and two-body levels. These operators are well defined but have different properties from those considered here; for example, even one-body operators flow. The in-medium SRG shows great promise as a microscopic method of deriving effective shell-model interactions for nuclei and the study of the corresponding operators is an important topic for future investigation.

In this article, we restrict our attention to the deuteron, which means only one- or two-body operators are relevant. Furthermore, any running with $s$ is due to an induced twobody part. In practice, working in the two-body system in the center-of-mass frame makes the occurrence of two-body operators uneventful; there are far more consequences for $A>2$. We defer to the sequel [10] the discussion of evolving and extracting operator components and embedding them in larger $A$ systems using a harmonic-oscillator basis.

Nuclear SRG studies to date have focused primarily on the calculation of binding energies and phase shifts to analyze the characteristics of the SRG-evolved interactions. A limited analysis of the deuteron momentum distribution defined by the initial operator $a_{q}^{\dagger} a_{q}$ has also been made [13] and decoupling of long-distance operators for the rms radius, quadrupole moment, and $r^{-1}$ have been examined [7], but only for the bare operators. We expand upon these studies here, emphasizing the nature of SRG-evolved operators and also include the first calculations of deuteron charge, quadrupole, and magnetic form factors. However, we use these electromagnetic operators as test cases for addressing questions about operator evolution and not yet for systematic comparison to experiment, which requires a more complete treatment of the $s=0$ operators.

Other issues arise about processes with large momentum transfers, such as $\left(e, e^{\prime} p\right)$. Theoretical analyses relate such experiments to nuclear momentum distributions if the impulse approximation is assumed to be valid for a high-cutoff interaction [15]. Calculations find nearly universal scaling of the highmomentum tails, which is interpreted in terms of short-range correlations in the nuclear wave functions. It might be thought naively that this physics is beyond the reach of low-momentum approaches, for which wave functions have drastically reduced short-range correlations. However, Eq. (7) is unequivocal: The experimental cross section is unchanged with SRG evolution to low momentum if no approximations are made, even if the evolved wave function has almost no short-range correlations. But does the calculation become intractable because the evolution of the momentum occupation operator makes it too complicated (e.g., strong nonlocalities, too-large manybody components)? We begin to address this question by showing that under the relevant kinematic conditions there is factorization of the unitary transformation $U_{s}$, which leads to significant simplifications and an alternative interpretation of the universal high-momentum dependence and scaling.

The plan of the article is as follows. In Sec. II, we explore whether decoupling of the Hamiltonian for two-body systems is mirrored in the operator flow, focusing on evolution of the momentum distribution as a characteristic example. In Sec. III, we consider the evolution of other operators, including electromagnetic form factors in the deuteron, which are all found to flow to smooth, low-momentum forms. Variational 


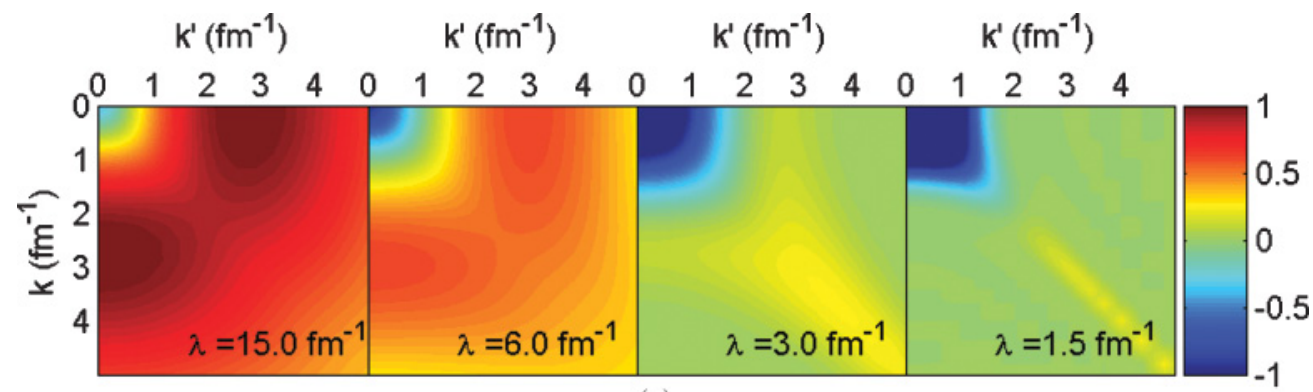

(a)

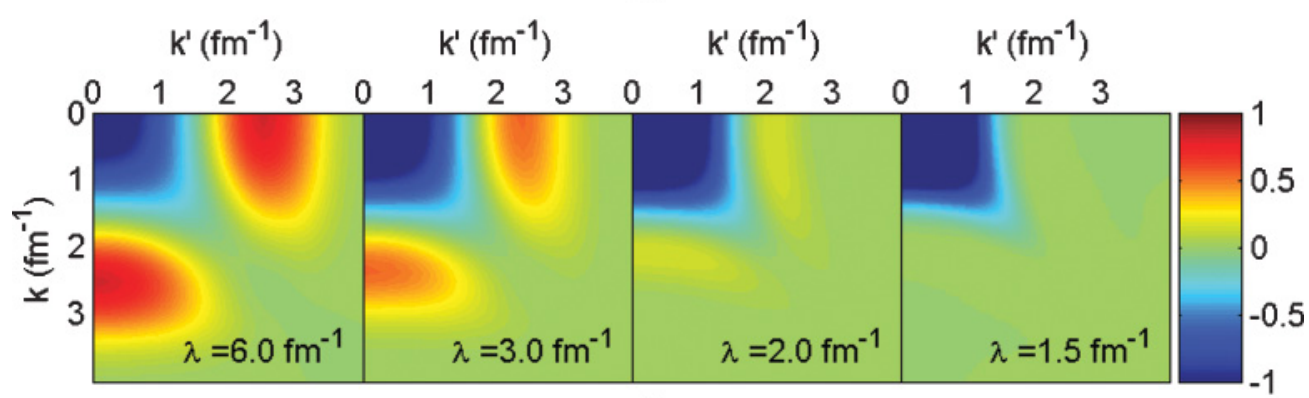

(b)

FIG. 1. (Color online) SRG evolution of the momentum-space ${ }^{3} S_{1}$ potential starting with (a) Argonne $v_{18}$ (AV18) from $\lambda=15 \mathrm{fm}{ }^{-1}$ to $\lambda=1.5 \mathrm{fm}^{-1}[16]$ and (b) $\mathrm{N}^{3} \mathrm{LO}(500 \mathrm{MeV})$ from $\lambda=6 \mathrm{fm}^{-1}$ to $\lambda=1.5 \mathrm{fm}^{-1}$ [17].

calculations of the evolved deuteron binding energy and operator matrix elements are explored in Sec. IV. The factorization of the unitary transformation operator under certain conditions is demonstrated in Sec. V, along with a model calculation of the momentum distribution for $A \geqslant 2$. Our conclusions are summarized in Sec. VI.

\section{DECOUPLING AND THE DEUTERON MOMENTUM DISTRIBUTION}

\section{A. Potentials and decoupling}

In this section, we specialize the SRG evolution to a two-particle partial-wave momentum basis with flow operator $G_{s}=T_{\text {rel }}$. The flow equation,

$$
\frac{d H_{s}}{d s}=\left[\left[T_{\mathrm{rel}}, H_{s}\right], H_{s}\right]=\frac{d V_{s}}{d s}
$$

(recall that $T_{\text {rel }}$ is chosen to remain constant), for nucleonnucleon $(N N)$ potentials is projected onto each channel using $1=\frac{2}{\pi} \int_{0}^{\infty} q^{2} d q|q\rangle\langle q|$, with $\hbar=M=1$, yielding

$$
\begin{aligned}
\frac{d V_{s}\left(k, k^{\prime}\right)}{d s}= & -\left(k^{2}-k^{\prime 2}\right)^{2} V_{s}\left(k, k^{\prime}\right) \\
& +\frac{2}{\pi} \int_{0}^{\infty} q^{2} d q\left(k^{2}+k^{\prime 2}-2 q^{2}\right) V_{s}(k, q) V_{s}\left(q, k^{\prime}\right) .
\end{aligned}
$$

This equation is implemented in a discretized Gaussian quadrature basis as a set of coupled differential equations for the matrix elements (angular momentum indices in the coupled channels have been suppressed) that are solved numerically.

The flow is visualized in Fig. 1 for two representative initial potentials, Argonne $v_{18}(\mathrm{AV} 18)$ [16] and a chiral $\mathrm{N}^{3} \mathrm{LO}$
$(500 \mathrm{MeV})$ effective field theory (EFT) potential from Ref. [17], which are currently the most commonly used $N N$ interactions for microscopic nuclear structure calculations. The figures show snapshots of the potential matrix $V_{s}\left(k, k^{\prime}\right)$ in the ${ }^{3} S_{1}$ partial wave at several values of $\lambda$, which is a useful alternative parameter to characterize the flow. It is related to $s$ by $\lambda=s^{-1 / 4}$. The scale at the right indicates the strength of the potential (in fm); Gaussian mesh weights are not shown. One can see that the large matrix elements between low and high momentum at the initial $\lambda$ 's shown (particularly for AV18) are suppressed by $\lambda=1.5 \mathrm{fm}^{-1}$; that is, we see decoupling for both potentials.

The decoupling seen in Fig. 1 is readily understood from the SRG flow equations. Because of the dominance of the kinetic energy, Eq. (10) for sufficiently off-diagonal $k$ and $k^{\prime}$ is given to good approximation by

$$
\frac{d V_{s}\left(k, k^{\prime}\right)}{d s} \approx-\left(k^{2}-k^{\prime 2}\right)^{2} V_{s}\left(k, k^{\prime}\right),
$$

which, when solved, predicts

$$
V_{s}\left(k, k^{\prime}\right) \approx e^{-s\left(k^{2}-k^{\prime 2}\right)^{2}} V_{s=0}\left(k, k^{\prime}\right)=e^{-\frac{\left(k^{2}-k^{\prime 2}\right)^{2}}{\lambda^{4}}} V_{\lambda=\infty}\left(k, k^{\prime}\right) .
$$

Thus, using this generator we can see that the far off-diagonal elements of the potential matrix are suppressed exponentially with an approximate width given by the flow parameter $\lambda$ [6]. (If the potential is plotted as a function of $k^{2}$, the width of the partially diagonalized potential is clearly seen to be well approximated by $\lambda^{2}[1]$.) While it is not evident from the figure, the potential is also very smooth.

It is not immediately clear, however, that this decoupling will be advantageous for the calculation of observables other than the binding energy. If we project the operator flow 
equation

$$
\frac{d \widehat{O}_{s}}{d s}=\left[\left[T_{\mathrm{rel}}, H_{s}\right], \widehat{O}_{s}\right]
$$

onto the partial wave momentum basis, we find

$$
\begin{aligned}
\frac{d O_{s}\left(k, k^{\prime}\right)}{d s}= & \frac{2}{\pi} \int_{0}^{\infty} q^{2} d q\left[\left(k^{2}-q^{2}\right) V_{s}(k, q) O_{s}\left(q, k^{\prime}\right)\right. \\
& \left.+\left(k^{\prime 2}-q^{2}\right) O_{s}(k, q) V_{s}\left(q, k^{\prime}\right)\right],
\end{aligned}
$$

and decoupling is not manifest. Further, it is not clear if $\lambda$ provides a measure of decoupling in the case of a general operator. So we turn to visualizations of the operator matrix elements for guidance. As noted earlier, in practice we do not solve the flow equation for operators, but apply the unitary transformation Eq. (4) at $s$ by first solving the inital and final Hamiltonians for the eigenvectors and constructing the matrix

$$
U_{s}\left(k_{i}, k_{j}\right)=\sum_{\alpha}\left\langle k_{i} \mid \psi_{\alpha}(s)\right\rangle\left\langle\psi_{\alpha}(0) \mid k_{j}\right\rangle,
$$

where $\left\{k_{i}\right\}$ is the discrete momentum mesh.

\section{B. Momentum distribution}

We begin our examination of operator evolution with perhaps the simplest nontrivial example: the momentum occupation operator $a_{q}^{\dagger} a_{q}$ in the center-of-mass frame (in this frame-for the $A=2$ particle space $-q$ is the magnitude of both the relative momentum and the single-particle momentum). By varying the momentum $q$, we can gain insight into how the SRG evolution behaves for initial operators dominated by either high or low momenta. In Fig. 2 the plot of the momentum distribution is reproduced from the expectation value $\left\langle\psi_{d}\left|a_{q}^{\dagger} a_{q}\right| \psi_{d}\right\rangle$ in the deuteron for the two initial potentials of Fig. 1. The solid line is the result when the unevolved wave function (i.e., the wave function derived from the unevolved potential) is used with the unevolved $a_{q}^{\dagger} a_{q}$ operator. This sets the baseline for evaluating the effects of the SRG for each potential. When one uses the evolved wave function with the evolved operator, $U_{s} a_{q}^{\dagger} a_{q} U_{s}^{\dagger}$, the lines are indistinguishable. The dashed and dot-dashed lines are calculated using the unevolved operator with the evolved wave function at $\lambda=2.0 \mathrm{fm}^{-1}$ and $\lambda=1.5 \mathrm{fm}^{-1}$, respectively. These curves quantify the effect of not consistently evolving operators and also give the momentum dependence of the evolved wave functions. As can be seen, the high-momentum components of the wave functions are significantly suppressed, as is consistent with the decoupling seen in the potential.

An analogous visual representation to that used for the potential allows us to analyze the RG flow and properties of these objects. Figures 3 and 4 illustrate the flow of the momentum occupation operator consistent with the renormalization of the $\mathrm{N}^{3} \mathrm{LO} 500-\mathrm{MeV}$ potential. We present the SRG results using only one potential, but qualitatively similar results will be obtained using any nuclear potential of interest. Each of the figures shows three sequences: the initial operator matrix elements $\left\langle k\left|a_{q}^{\dagger} a_{q}\right| k^{\prime}\right\rangle$ evolved to four different $\lambda=s^{-1 / 4}$ values and the integrand of $\left\langle\psi_{d}(s)\left|\left(a_{q}^{\dagger} a_{q}\right)_{s}\right| \psi_{d}(s)\right\rangle$ with first linear and then logarithmic scales. The operators shown correspond to those used to calculate the momentum distribution at $q=0.34 \mathrm{fm}^{-1}$ and $q=3.02 \mathrm{fm}^{-1}$ [marked by the dotted lines in Fig. 2(b)]. It is apparent that the unevolved operator is simply a $\delta$ function in momentum space (some minor evolution can already be seen at $\lambda=6 \mathrm{fm}^{-1}$ because the scale must be magnified to view the evolution at lower values of $\lambda$ ).

Consider the operator (top row) sequences first. For both $q$ values, the evolution begins along the momentum axes around the $\delta$ function where we see strength developing that was not present in the original operator. This behavior can be understood from the momentum basis SRG [Eq. (14)] and the features of the corresponding potential evolution. Holding $k$ fixed, one can see that only the second term in the integral initially picks up strength along the axis that passes through

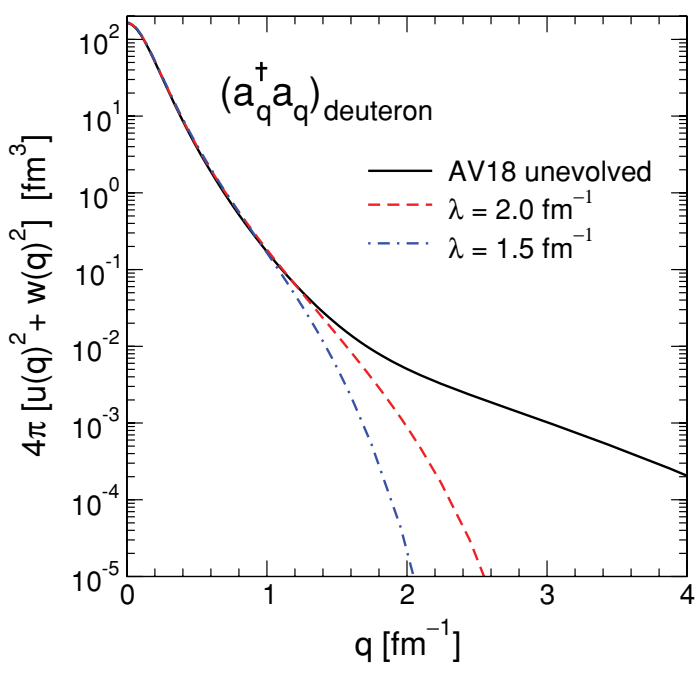

(a)

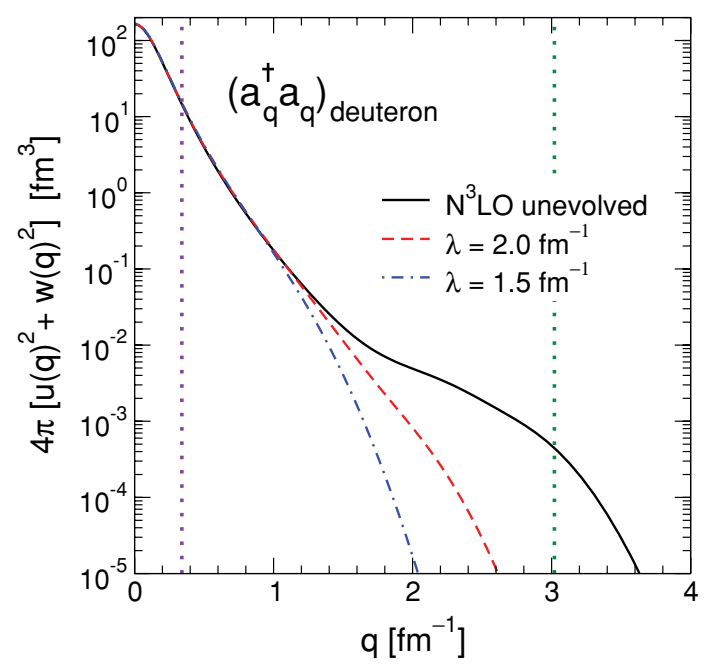

(b)

FIG. 2. (Color online) The momentum distribution in the deuteron as given by the expectation value of the bare number operator $a_{q}^{\dagger} a_{q}$ for the (a) Argonne $v_{18}$ (AV18) [16] and (b) $\mathrm{N}^{3} \mathrm{LO}(500 \mathrm{MeV})$ [17] potentials, both evolved and unevolved. 


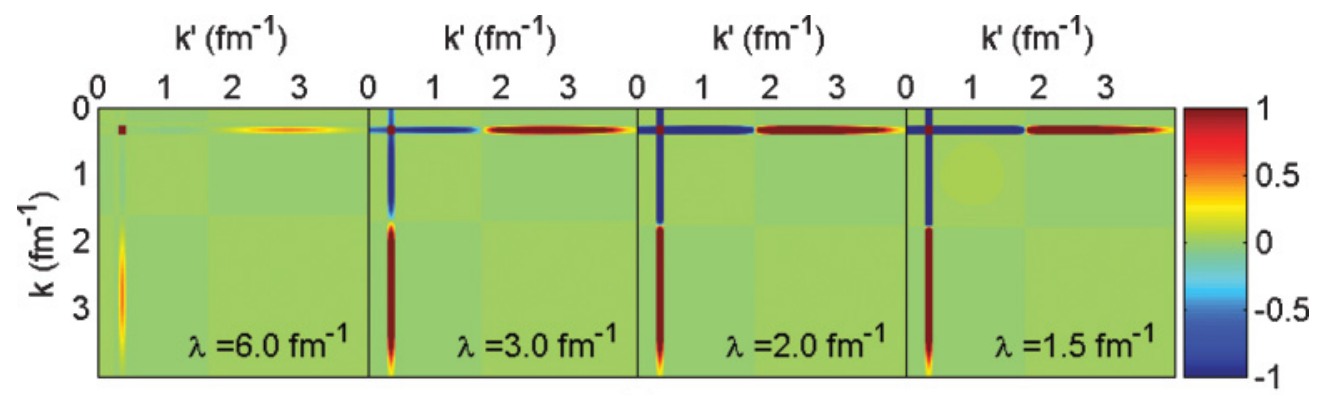

(a)

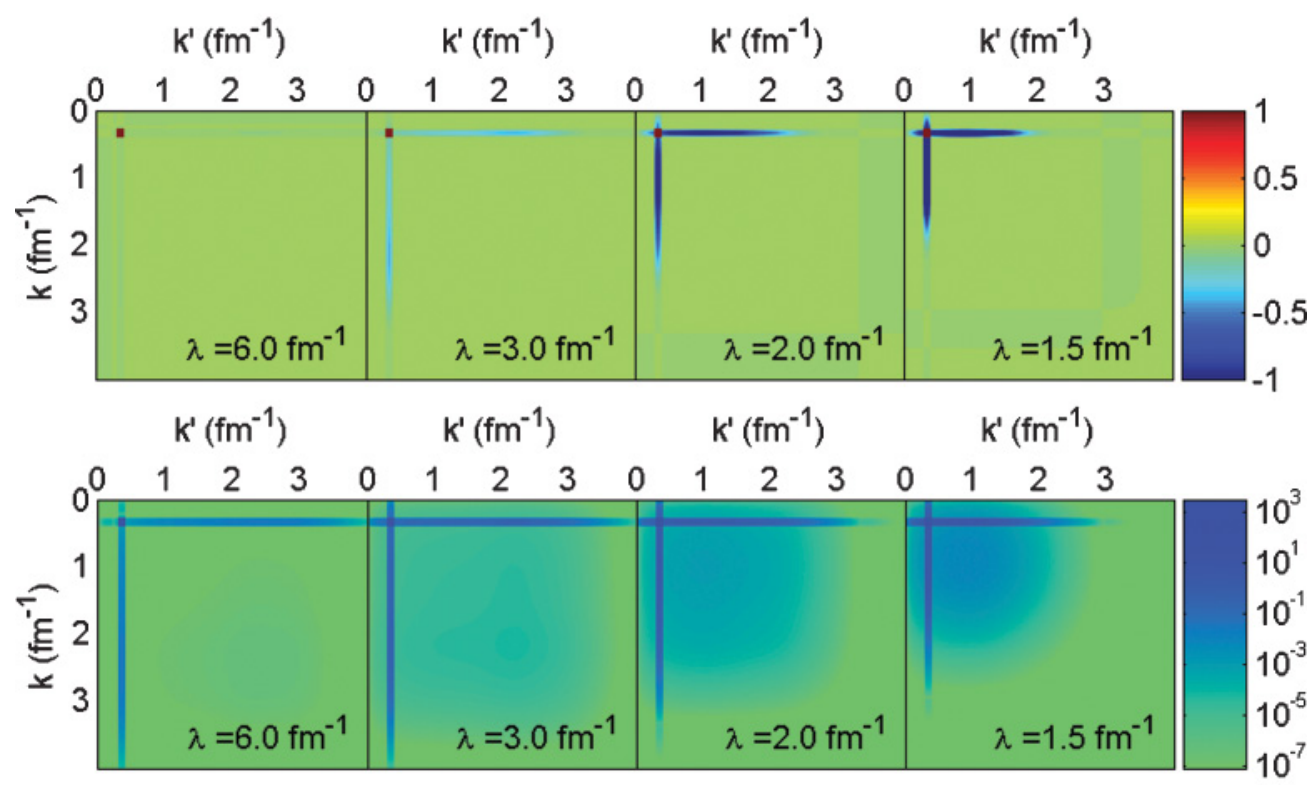

(b)

FIG. 3. (Color online) (a) SRG evolution of the operator $\left\langle k\left|a_{q}^{\dagger} a_{q}\right| k^{\prime}\right\rangle$ for $q=0.34 \mathrm{fm}^{-1}$ in the ${ }^{3} S_{1}$ partial wave from $\lambda=6 \mathrm{fm}{ }^{-1}$ to $\lambda=1.5 \mathrm{fm}^{-1}$, with the $\mathrm{N}^{3} \mathrm{LO}(500 \mathrm{MeV})$ [17] initial potential. (b) Integrand of $\left\langle\psi_{d}(s)\left|\left(a_{q}^{\dagger} a_{q}\right)_{s}\right| \psi_{d}(s)\right\rangle$ with linear (top) and logarithmic magnitude (bottom) scales.

the $\delta$ function, because the operator is zero everywhere else, and vice versa holding $k^{\prime}$ fixed. For the operator at high $q$ we see that it develops more and more strength at low momentum as it evolves. The need for this additional strength is particularly evident because of the decoupling via evolution of the potential and the consequent suppression of highmomentum components in the deuteron wave function, as seen in Fig. 2(b). As a result, the operator must pick up additional strength for the expectation values to remain unchanged. This strength can appear in two ways: (i) It can come in at low momentum, as we see here; or (ii) the operator can gain strength only at high momenta, where the operator would have to become pathologically large. If the second case were to occur, practical calculations with the SRG in a reduced basis would not be possible. This is found empirically to not be the case, and we can be confident such pathologies will not occur based on more general arguments discussed in what follows.

The operator at low $q$, however, picks up some strength at larger values of momentum than present in the initial operator. This is also needed to compensate for the suppression of low momentum dependence in high-energy eigenstates to maintain their expectation values. One should note that the operator display scale used here can be a bit deceptive in that it has been amplified to make the qualitative features of the evolution more apparent. Most of the evolution does, in fact, remain at low momentum for deuteron expectation values.

We show the occupation operator as an integrand given by $\left\langle\psi_{d}\left|a_{q}^{\dagger} a_{q}\right| \psi_{d}\right\rangle$ in Figs. 3(b) and 4(b). The expectation value filters the general operator by weighting its matrix elements with the deuteron wave function. Now we can see a clean RG flow in the strength for both operators. The integrand of the operator at $q=3.02 \mathrm{fm}^{-1}$ begins as a sharp spike, corresponding to the original operator, but then flows out along the momentum axes to lower momentum. By the time the integrand reaches lower values of $\lambda$ in the evolution, nearly all of the strength in the expectation value is in the low-momentum region. The original spike disappears as the wave function dependence at high momentum falls off.

As for the operator at $q=0.34 \mathrm{fm}^{-1}$, the strength does begin to flow out to some extent but remains almost entirely in the low-momentum region. Once again, the display scale has overemphasized the extent of the evolution in the values of the integrand. The spike that remains at $\lambda=1.5 \mathrm{fm}^{-1}$ actually contains $\approx 96 \%$ of the full expectation value. Owing 


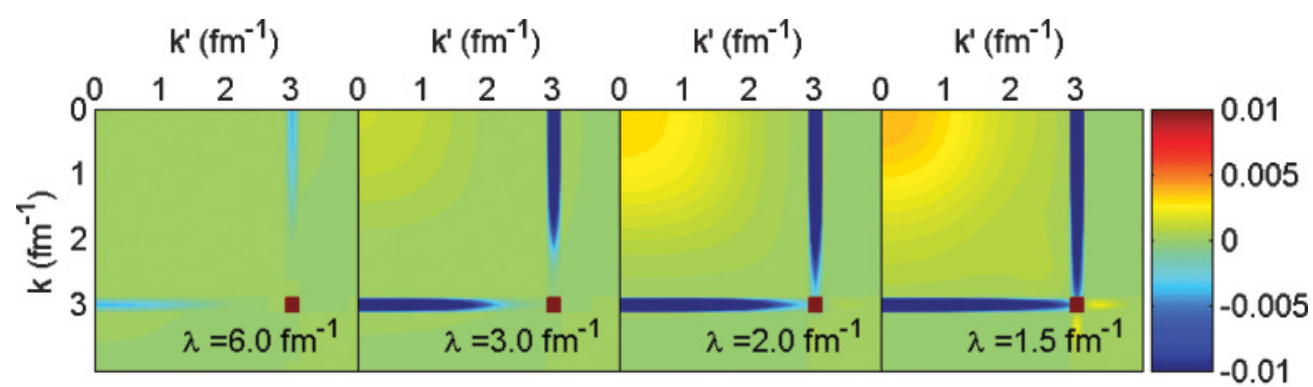

(a)

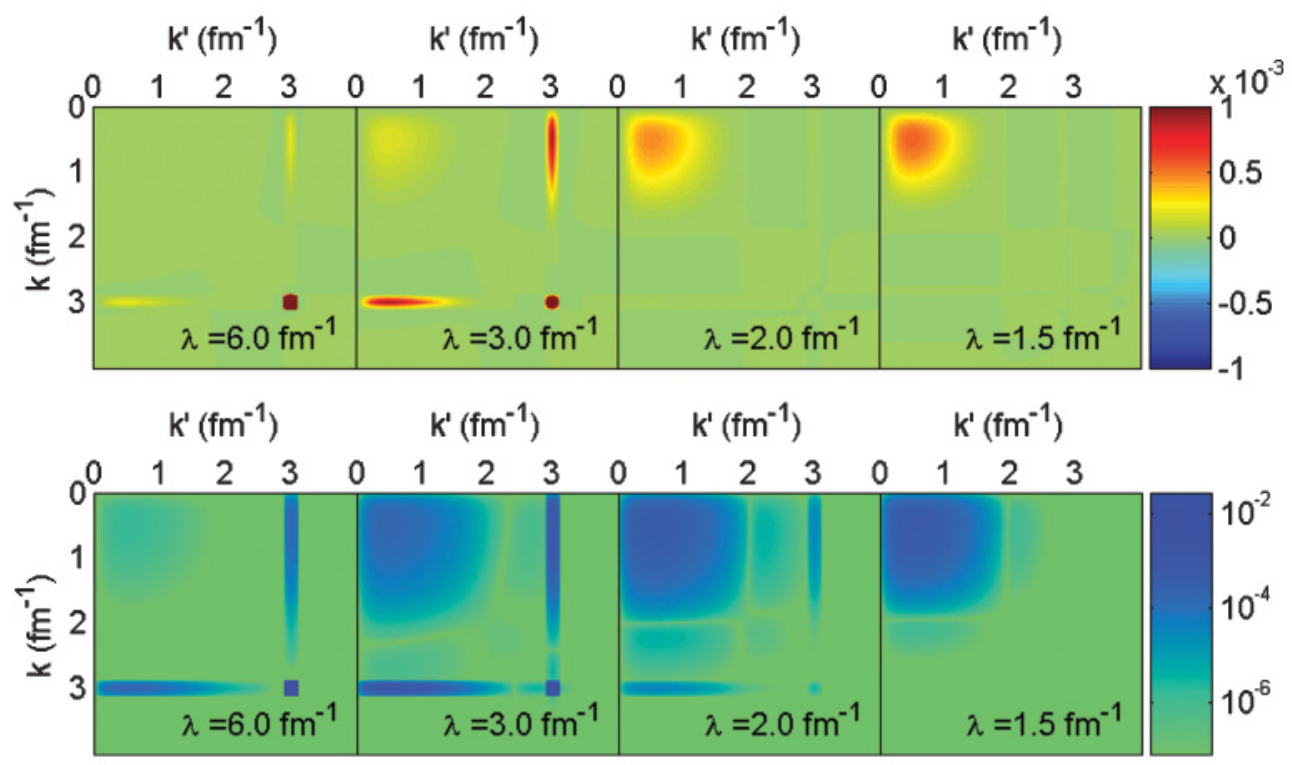

(b)

FIG. 4. (Color online) Same as Fig. 3 but for $q=3.02 \mathrm{fm}^{-1}$.

to the possibility of misinterpreting these plots on a linear display scale, we also include the same plots with logarithmic display scale. These pictures show only the magnitude of the integrands but display nearly the full range of their values. Now it is conclusive that the strength of the high-momentum operator flows to low momentum, and the strength of the low-momentum operator remains at low momentum for a low-energy state. We see this pattern repeat in the calculation of other operators in the next section.

Despite the apparent changes in the integrands as they evolve, it is important to note that the sum of all the points (the expectation value) remains unchanged because of the unitarity of the SRG transformation. The momentum distribution calculations shown in Fig. 2 were performed in the full momentum space of the original potential. Decoupling of the potential allows us to truncate the model space, thereby making numerical simulations more feasible, while at the same time allowing us to calculate the correct binding energies. If the calculation of other expectation values must be performed in the full model space, then the benefits of the SRG would be lost. However, the redistribution of strength implies that we have a form of decoupling for the operator. A critical test is to verify that decoupling is maintained in the calculation of operator expectation values.
This check is shown in Figs. 5 and 6 for the momentum distributions of AV18 and $\mathrm{N}^{3} \mathrm{LO}$ potentials. To perform the check, we evolved the original Hamiltonians and operators in the full momentum space to various $\lambda$, then truncated the model space to $\Lambda$. The deuteron wave function derived in this truncated space is used to calculate the expectation value of the evolved number operators to produce the momentum distributions shown here. The figures show that when the SRG evolution $\lambda>$ $\Lambda$, the curves deviate significantly from that produced in the full space (because the wave function is distorted). However, once the operators are evolved to $\lambda$ below the truncation at $\Lambda$, the expectation values are reproduced for all values of momenta, even in the region outside of the new model space. Thus, decoupling is successful and $\lambda$ provides a rough guide as to where this decoupling occurs. We see that this is also the case for other operator matrix elements of interest, as well as understand further how this comes about, in what follows.

\section{General analysis}

The plots of the deuteron integrands show that no pathologies appear at high momentum in the evolved operators and verify that decoupling can be successful when calculating expectation values of this operator. The plots even indicate 


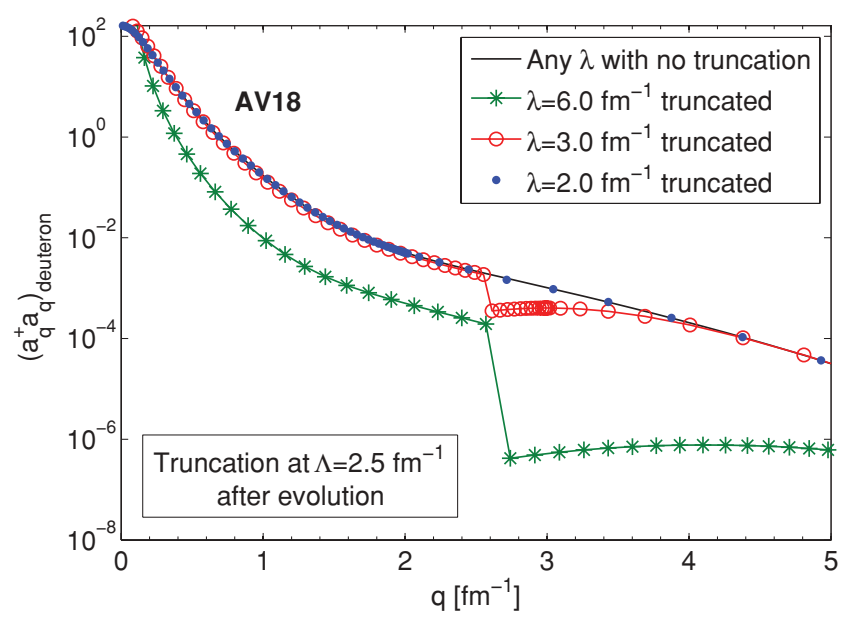

FIG. 5. (Color online) Decoupling in operator matrix elements is tested by calculating the momentum distribution in the deuteron after evolving the AV18 potential to several different $\lambda$ and then truncating the Hamiltonian and evolved occupation operators (i.e., set them to zero above $\Lambda=2.5 \mathrm{fm}^{-1}$ ).

where the model space can be truncated - this is simply where the integrand strength becomes negligible in the logarithmic plot. Here we develop a more general understanding of operator evolution to build confidence that pathologies will not occur in other operators.

Consider the representation of a generic operator in terms of the energy eigenstates,

$$
\widehat{O}_{s}=\sum_{i j} O_{i j}\left|\psi_{i}(s)\right\rangle\left\langle\psi_{j}(s)\right|,
$$

where

$$
O_{i j}=\left\langle\psi_{i}(s)\left|\widehat{O}_{s}\right| \psi_{j}(s)\right\rangle .
$$

It is important to remember that these matrix elements are invariant under SRG transformations, so $O_{i j}$ does not

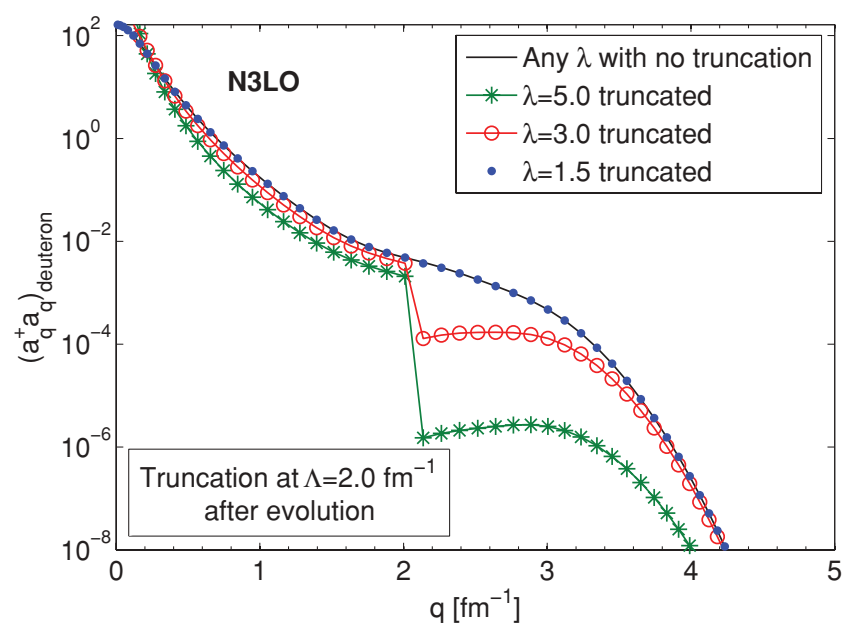

FIG. 6. (Color online) Decoupling in operator matrix elements is tested by calculating the momentum distribution in the deuteron after evolving the $\mathrm{N}^{3} \mathrm{LO}$ potential to several different $\lambda$ and then truncating the Hamiltonian and evolved occupation operators (i.e., set them to zero above $\Lambda=2.0 \mathrm{fm}^{-1}$ ). depend on $s$. Thus, the momentum-space behavior of evolved operators is given, in turn, by the momentum-space behavior of the evolved eigenstates - specifically, the sum of their outer products weighted by $O_{i j}$,

$$
O_{s}\left(k, k^{\prime}\right)=\sum_{i j} O_{i j}\left\langle k \mid \psi_{i}(s)\right\rangle\left\langle\psi_{j}(s) \mid k^{\prime}\right\rangle .
$$

The behavior of these eigenstates is well under control. As we have seen from the momentum distribution of the deuteron, low-energy bound-state wave functions are suppressed at high momentum. The rest of the (positive) eigenstates are effectively smeared out $\delta$ functions (normalized in a finite basis), which because of decoupling in the Hamiltonian become increasingly narrow peaks with the evolution in $s$. So, the evolution will not become pathological unless the unevolved operator is already pathological (i.e., only if some $O_{i j}$ are unnaturally large).

If we now consider this operator in the deuteron eigenstate $\left|\psi_{d}(s)\right\rangle=\left|\psi_{1}(s)\right\rangle$, we find that only the $O_{11}$ matrix element of the operator is projected out and the momentum dependence is given by the outer product of the deuteron wave function. Specifically, the only nonzero part of the operator will be formally given by

$$
O_{s}\left(k, k^{\prime}\right) \rightarrow O_{11}\left\langle k \mid \psi_{1}(s)\right\rangle\left\langle\psi_{1}(s) \mid k^{\prime}\right\rangle .
$$

However, if we would like to reconsider the issue of decoupling and a finite model space truncation, we find that we are restricted by the potential breaking of eigenstate orthogonality in the truncated space, that is, the extent to which

$$
\frac{2}{\pi} \int_{0}^{\Lambda} k^{2} d k\left\langle\psi_{i}(s) \mid k\right\rangle\left\langle k \mid \psi_{j}(s)\right\rangle \neq 0 \quad \text { for } i \neq j .
$$

The logarithmic integrand plots show us that this problem is negligible if one of the states is the deuteron. Furthermore, SRG-driven decoupling of states well separated in energy will make them increasingly orthogonal in the truncated space. Thus, the momentum-space evolution of operators in a specific basis can be brought under control; we will consider the case of induced many-body components of the operators in a sequel to this article [10].

\section{OTHER OPERATORS}

\section{A. Long-distance operators: $\left\langle r^{2}\right\rangle,\left\langle Q_{d}\right\rangle,\left\langle r^{-1}\right\rangle$}

We begin our presentation of additional operators with the evolution of operators for three paradigmatic expectation values in the deuteron: the rms radius, the quadrupole moment, and $r^{-1}$. These operators all act on relatively long distance scales. At leading order they are naturally defined in coordinate space so that deuteron expectation values can be written as [18]

$$
\begin{aligned}
\left\langle r_{d}\right\rangle & =\frac{1}{2}\left[\int_{0}^{\infty} d r r^{2}\left[u(r)^{2}+w(r)^{2}\right]\right]^{1 / 2}, \\
\left\langle Q_{d}\right\rangle & =\frac{1}{20} \int_{0}^{\infty} d r r^{2} w(r)[\sqrt{8} u(r)-w(r)],
\end{aligned}
$$

and

$$
\left\langle\frac{1}{r}\right\rangle=\int_{0}^{\infty} d r\left(\frac{1}{r}\right)\left[u(r)^{2}+w(r)^{2}\right]
$$




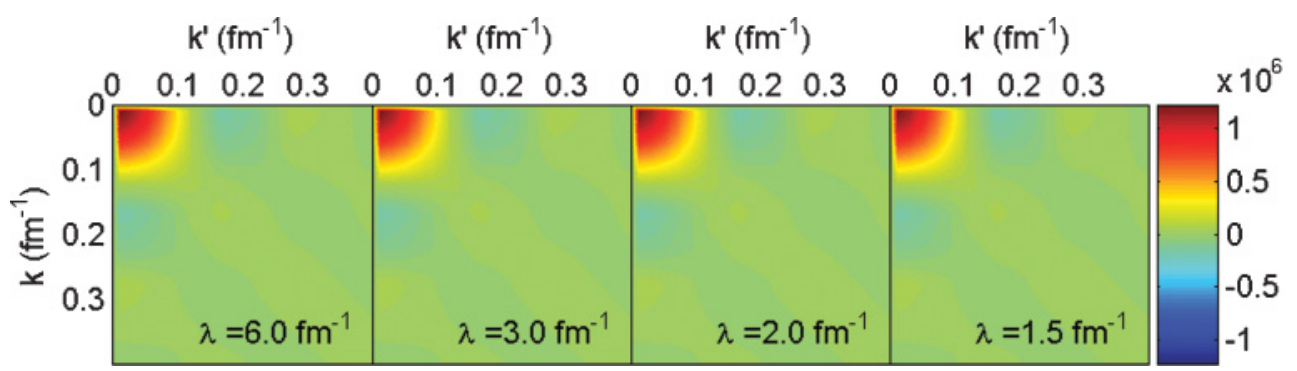

FIG. 7. (Color online) Operator evolution of $\left\langle k\left|r^{2}\right| k^{\prime}\right\rangle$ in the ${ }^{3} S_{1}$ partial wave from $\lambda=6 \mathrm{fm}^{-1}$ to $\lambda=1.5 \mathrm{fm}^{-1} \mathrm{using}$ the $\mathrm{N}^{3} \mathrm{LO}(500-\mathrm{MeV})$ potential.

where $u$ and $w$ are the ${ }^{3} S_{1}$ and ${ }^{3} D_{1}$ deuteron radial wave functions. However, we will continue to analyze the operators in momentum space. To avoid numerical instabilities associated with putting the derivatives in the momentumspace expressions on a mesh (see Ref. [18]), we extract the coordinate-space operators from Eqs. (21)-(23) and transform to the partial wave-momentum basis. For example, from Eq. (21) we can see that the $r^{2}$ operator is given by the diagonal matrix (discretized in coordinate space)

$$
\left\langle r\left|r^{2}\right| r^{\prime}\right\rangle=r^{2} \delta\left(r-r^{\prime}\right)
$$

in the ${ }^{3} S_{1}$ and ${ }^{3} D_{1}$ channels. The only transformations needed are given by

$$
\left\langle r \mid k ;{ }^{3} S_{1}\right\rangle=\sqrt{\frac{2}{\pi}} r k^{2} j_{0}(k r)
$$

and

$$
\left\langle r \mid k ;{ }^{3} D_{1}\right\rangle=\sqrt{\frac{2}{\pi}} r k^{2} j_{2}(k r),
$$

where the $j_{l}$ 's are spherical Bessel functions (additional partial waves are needed for states other than the deuteron, of course). The transformations are represented as $n \times m$ matrices (where $n$ and $m$ are the sizes of the coordinate- and momentum-space meshes, respectively) and applied to both sides of the $n \times n$ coordinate-space operator matrix to produce an $m \times m$ matrix in momentum space for each partial wave. Then, to evolve the operator in momentum space, we apply the unitary transformation $\widehat{O}_{s}=U_{s} \widehat{O} U_{s}^{\dagger}$.

The SRG evolution sequence for $r^{2}$ shown in Fig. 7 is again a picture of just the operator in the momentum basis. Note the restricted momentum scale of the plot, as well as the magnitude of the operator display scale. As a long-distance operator, the strength is highly concentrated at low momentum. It is evident that very little renormalization occurs at the lowest values of momentum, which is consistent with the findings for the number operator. Looking at the deuteron integrand of the $r^{2}$ operator in Fig. 8, the linear display scale plot shows the same behavior. The log scale shows the entirety of the contributions to the integral. At low momentum, the strength is orders of magnitude greater than elsewhere. While the pattern of the contribution changes very little with $\lambda$ in the linearly scaled plot, the log-scale plot clearly shows the characteristic exponential suppression with decreasing $\lambda$ due to the decoupling of the potential and consequent reduction of high-momentum components in the deuteron wave function.
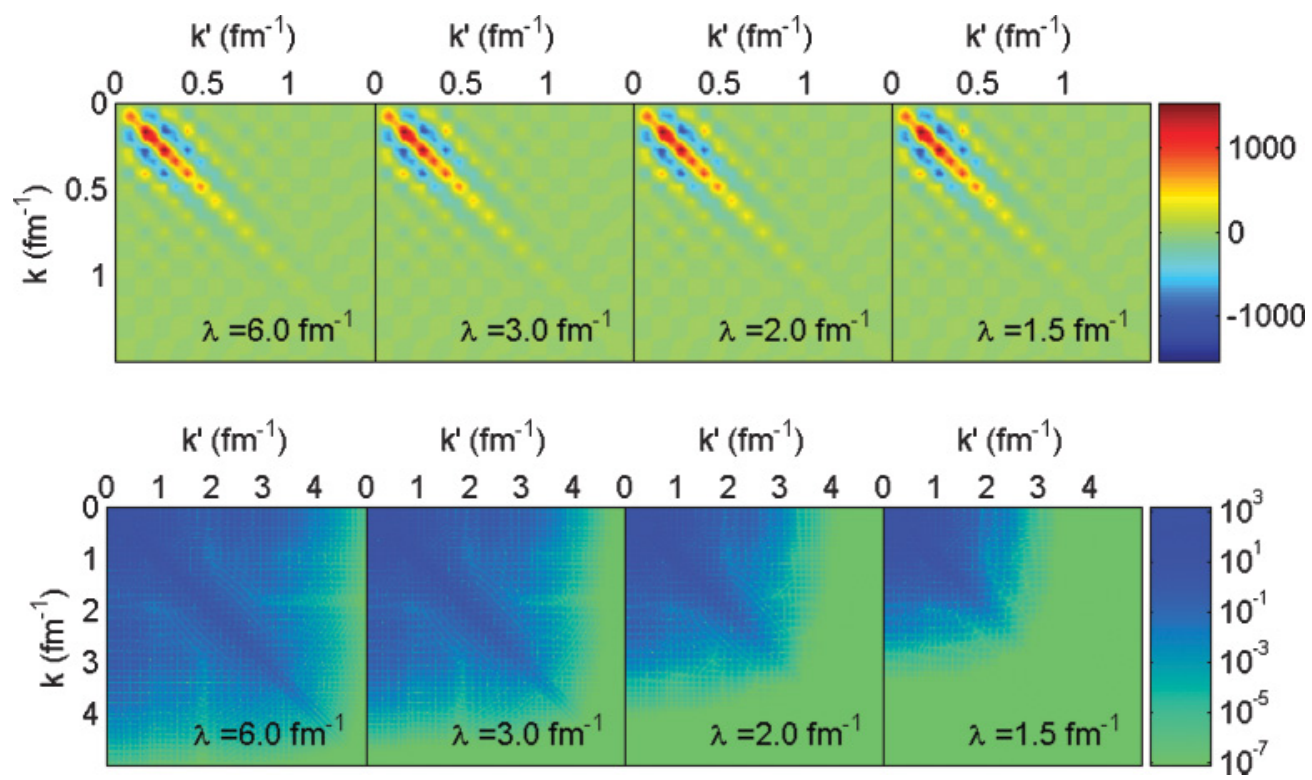

FIG. 8. (Color online) Integrand given by $\left\langle\psi_{d}\left|r^{2}\right| \psi_{d}\right\rangle$ and evolved from $\lambda=6 \mathrm{fm}^{-1}$ to $\lambda=1.5 \mathrm{fm}^{-1}$ using the N3LO 500-MeV potential with a linear color scale (top) and a logarithmic scale of the magnitude (bottom). Notice the difference in momentum scales. 

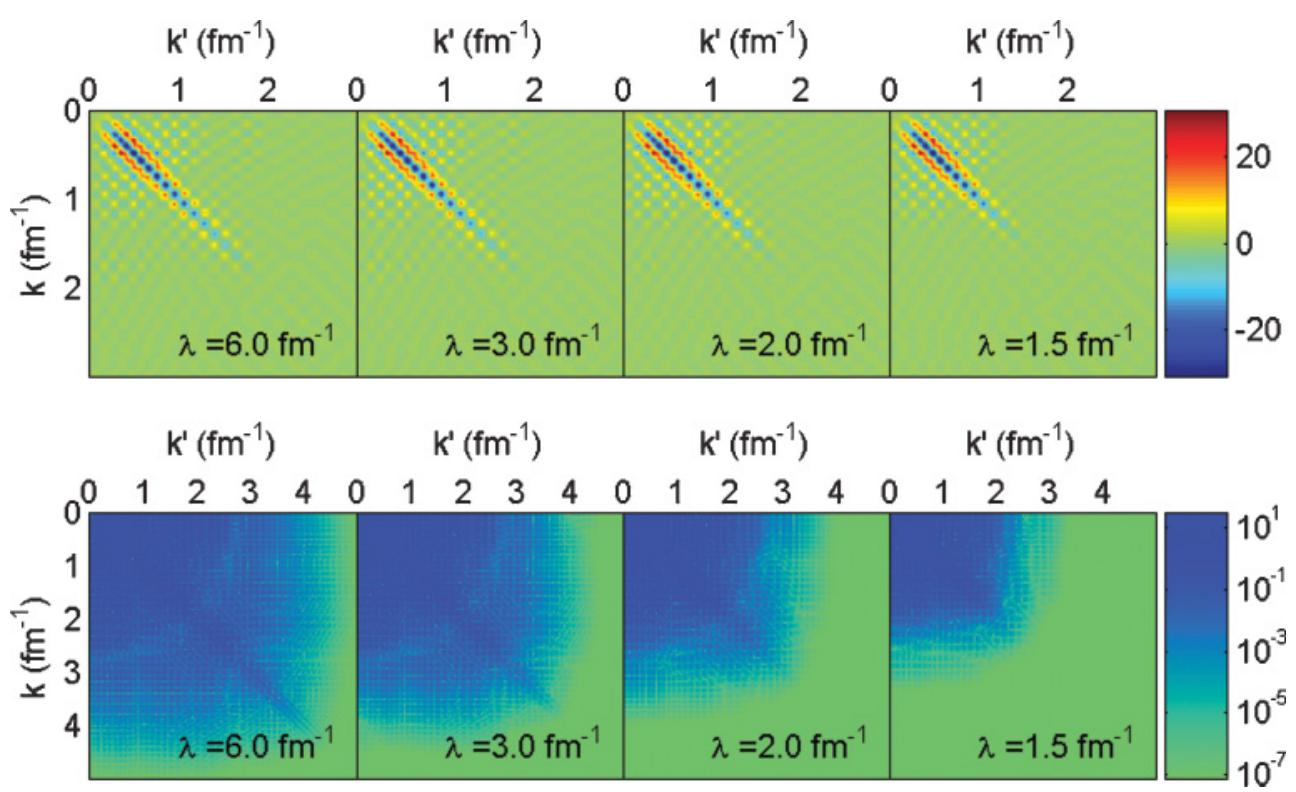

FIG. 9. (Color online) Integrand of the quadrupole moment expectation value in the deuteron and evolved from $\lambda=6 \mathrm{fm}^{-1}$ to $\lambda=1.5 \mathrm{fm}^{-1}$ using the N3LO 500-MeV potential with a linear color scale (top) and a logarithmic scale of the magnitude (bottom). Notice the difference in momentum scales.

The quadrupole moment operator is also a long-distance operator and we find its evolution shares most of the same characteristics found for the evolution of the $r^{2}$ operator (note that it also picks up strength in the ${ }^{3} S_{1}-{ }^{3} D_{1}$ channel). The deuteron integrand is shown in Fig. 9. There is little change in the actual evolution of the operator, while highmomentum contributions become exponentially suppressed. The lack of evolution can be quantified by calculating the expectation value of the unevolved operator with the evolved wave function. Results for various $\lambda$ are given in Table I. Note that we are using the basic, one-body quadrupole moment operator without two-body or other higher-order renormalized corrections. The "true" value for this potential and operator is $Q_{d} \approx 0.275 \mathrm{fm}^{2}$ while the experimental value of this quantity is $Q_{\exp } \approx 0.285 \mathrm{fm}^{2}$. Thus, the induced two-body contribution is the same order as omitted two-body contributions to the initial operator. As such, the SRG has not led to any changes larger than one would expect from including the fully renormalized operator from the EFT.

Finally, in Fig. 10 we show the evolution in the deuteron of the $r^{-1}$ operator, which has larger contributions at short range than the previous examples. Consequently, this operator is more spread out in momentum space. Yet we see the same general behavior with respect to renormalization and the suppression at large momenta without, as usual, any changes in the expectation value.

TABLE I. Expectation value of the quadrupole moment given by the unevolved operator with the evolved deuteron wave function.

\begin{tabular}{lcccc}
\hline \hline$\lambda$ & $6.0 \mathrm{fm}^{-1}$ & $3.0 \mathrm{fm}^{-1}$ & $2.0 \mathrm{fm}^{-1}$ & $1.5 \mathrm{fm}^{-1}$ \\
\hline$Q_{d}$ & $0.275 \mathrm{fm}^{2}$ & $0.274 \mathrm{fm}^{2}$ & $0.269 \mathrm{fm}^{2}$ & $0.260 \mathrm{fm}^{2}$ \\
\hline \hline
\end{tabular}

\section{B. Deuteron form factors: $G_{\mathrm{C}}, G_{\mathrm{Q}}$, and $G_{\mathrm{M}}$}

We now turn to the SRG evolution of electromagnetic operators that determine the deuteron charge, quadrupole, and magnetic form factors (i.e., $G_{\mathrm{C}}, G_{\mathrm{Q}}$, and $G_{\mathrm{M}}$, respectively) [19-23]. We restrict our discussion to deuteron expectation values that have been derived consistently with chiral EFT at leading order in coordinate space [23]. These are given by

$$
\begin{aligned}
G_{\mathrm{C}}\left(Q^{2}\right)= & G_{\mathrm{E}}^{(s)}\left(Q^{2}\right) \int d r\left[u^{2}(r)+w^{2}(r)\right] j_{0}(|\mathbf{q}| r / 2), \\
G_{\mathrm{Q}}\left(Q^{2}\right)= & G_{\mathrm{E}}^{(s)}\left(Q^{2}\right) \frac{6 \sqrt{2}}{Q^{2}} \\
& \times \int d r\left[u(r) w(r)+w^{2}(r) / \sqrt{8}\right] j_{2}(|\mathbf{q}| r / 2), \\
G_{\mathrm{M}}\left(Q^{2}\right)= & G_{\mathrm{E}}^{(s)}\left(Q^{2}\right) \frac{3}{2} \int d r w^{2}(r)\left[j_{0}(|\mathbf{q}| r / 2)+j_{2}(|\mathbf{q}| r / 2)\right] \\
& +G_{\mathrm{M}}^{(s)}\left(Q^{2}\right) 2 \int d r u^{2}(r) j_{0}(|\mathbf{q}| r / 2) \\
& +G_{\mathrm{M}}^{(s)}\left(Q^{2}\right)\left\{\sqrt{2} \int d r u(r) w(r) j_{2}(|\mathbf{q}| r / 2)\right. \\
& \left.-\int d r w^{2}(r)\left[j_{0}(|\mathbf{q}| r / 2)-j_{2}(|\mathbf{q}| r / 2)\right]\right\}
\end{aligned}
$$

where $Q^{2}=|\mathbf{q}|^{2}$ and $G_{\mathrm{E}}^{(s)}\left(Q^{2}\right)$ and $G_{\mathrm{M}}^{(s)}\left(Q^{2}\right)$ are the singlenucleon isoscalar electric and magnetic form factors obtained from the parametrization given in Ref. [24]. From these coordinate-space expressions, we can apply the same procedure used earlier to extract the operators, then use Eqs. (25) and (26) to convert to momentum space and transform via the SRG unitary transformation. One should note that starting from a coordinate-space operator is by no means essential; it is simply a numerical convenience in this case. 


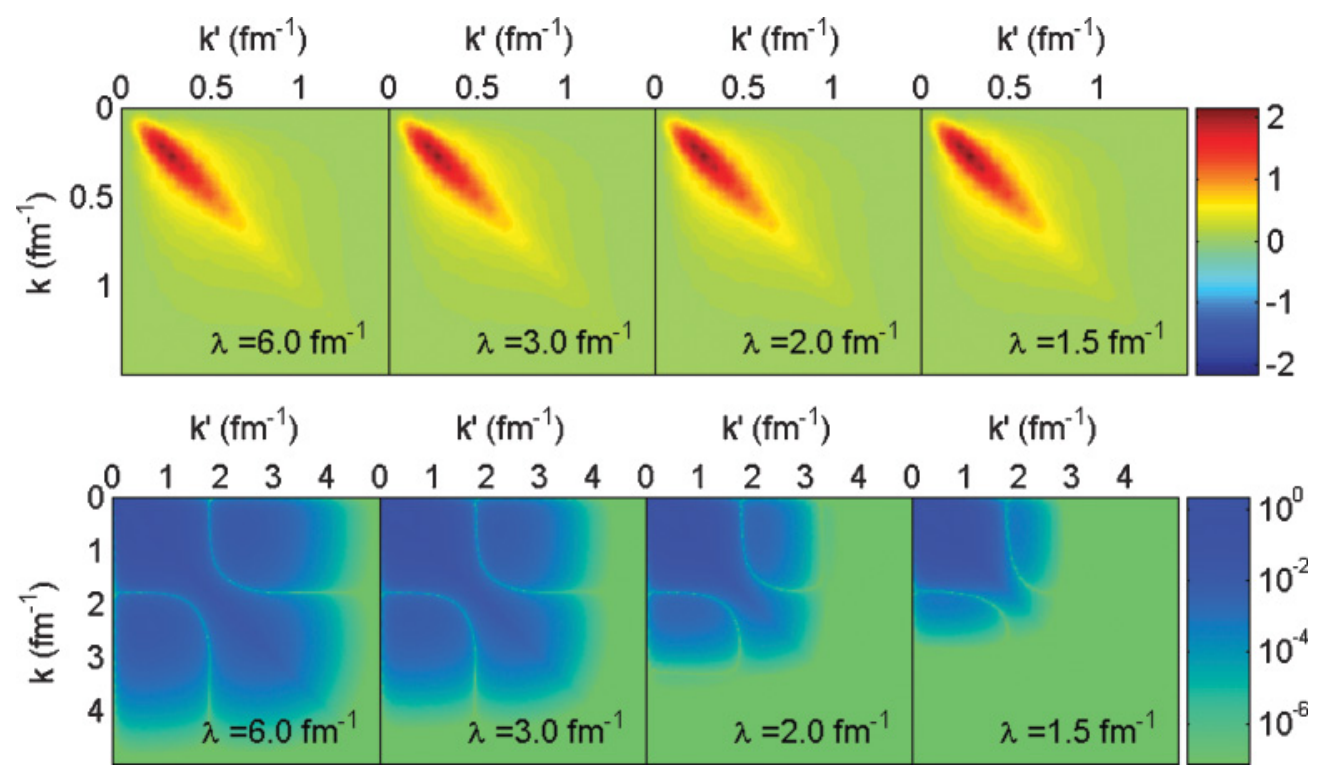

FIG. 10. (Color online) Integrand given by $\left\langle\psi_{d}\left|r^{-1}\right| \psi_{d}\right\rangle$ and evolved from $\lambda=6 \mathrm{fm}^{-1}$ to $\lambda=1.5 \mathrm{fm}^{-1}$ using the N3LO 500-MeV potential with a linear color scale (top) and a logarithmic scale of the magnitude (bottom). Notice the difference in momentum scales.

The expectation values as functions of $q=\sqrt{Q^{2}}$ for each of these operators is presented in Fig. 11. The solid line has been calculated using the unevolved potential with the unevolved operator, which again serves as our reference value. The starred points are calculated using the evolved wave function with the evolved operator (both at $\lambda=1.5 \mathrm{fm}^{-1}$ ). As advertised, they lie precisely on top of the solid line for all values of $q$, up to small numerical errors. The dot-dashed line is calculated using the unevolved operator with the evolved wave function as an indication of the effect of renormalization on the expectation value. We see noticeable deviation above $q \sim \lambda$; however, from the magnitude of the suppression seen in the wave function at high momentum, one might have expected the curve to drop much faster with respect to $q$. However, the form factor operators probe momenta in the deuteron center-of-mass frame, whereas $q$ is specified in the laboratory frame. Thus, the operators are probing the wave function largely at $\frac{1}{2} q$, which again brings the calculations in line with our SRG expectations.

The basic features of the SRG evolution of all three operators are qualitatively very similar, so we present the visual matrix representation of the magnetic form factor at high and low $q$ as a representative example in Figs. 12 and 13 . This form factor picks up strength in all deuteron channels. The high-momentum form factor has a much greater diffusion of strength at high momentum than seen in any of the static properties explored earlier. Yet it is apparent that the strength in the operator flows to low momentum in this case also. In contrast, the low-momentum operator exhibits very little renormalization, as we have come to expect. For both cases, we can see from the logarithmic display scale plots that the momentum dependence of the form factors is virtually eliminated at large momenta without affecting the outcome of the computation.

This lack of dependence on high momenta in evaluating expectation values is particularly significant for the practical application of the SRG in calculations of low-energy few- or many-body systems. Had this renormalization led to singular behavior in the operators at high momentum, the effects due to the suppression of the wave function would have been negated and led to wildly erroneous results in the evaluation of observables in a reduced model space. The arguments in Sec. II C explain why this will generally be the case.

\section{VARIATIONAL CALCULATIONS}

We have argued previously that SRG-evolved interactions and the resulting wave functions become "simpler." Variational calculations of the ground-state wave function can provide a test (and additional meaning) of the extent to which this is true in practical applications. The decoupling of high- and low-momentum states caused by SRG evolution means that the resulting wave functions are much less correlated than the original wave functions. Consequently, one expects that variational calculations of the evolved wave functions should be effective with a much simpler ansatz than one would normally require for the corresponding unevolved interaction. In conjunction with this, one might be concerned that a delicate interplay of the evolved operators and wave functions would be necessary to preserve matrix elements. However, it turns out that the evolved operators are not only equally good, but actually superior to the original operators in this respect. Rather than high-momentum operators picking up small pieces of the wave function (which could never be reproduced by a simple variational calculation), we get a smooth sum over where the wave function is large and easily approximated. We illustrate these points here by choosing a simple variational ansatz and looking at how convergence improves for SRGevolved operator expectation values.

Variational calculations have been performed on lowmomentum potentials (specifically " $V_{\text {low } k}$," derived using an 


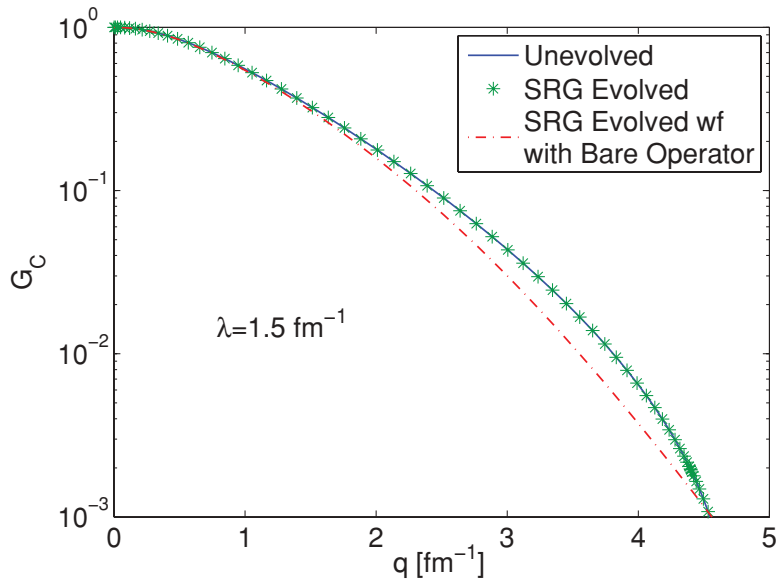

(a)

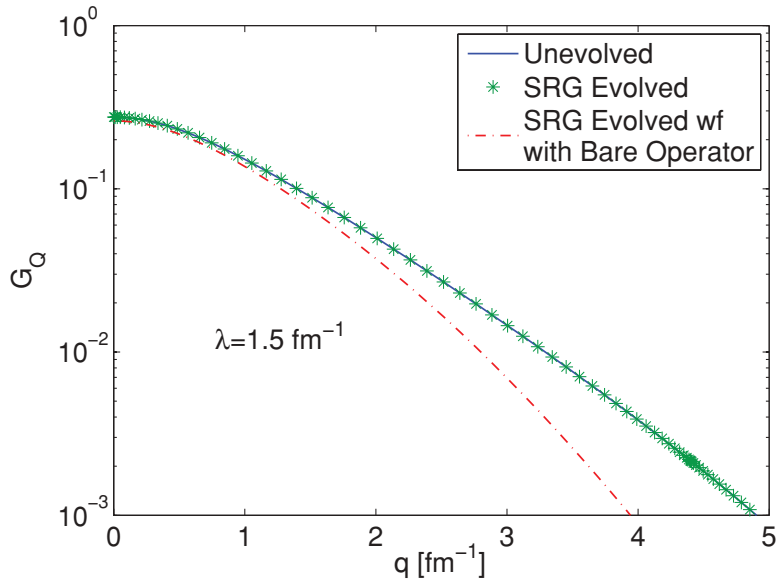

(b)

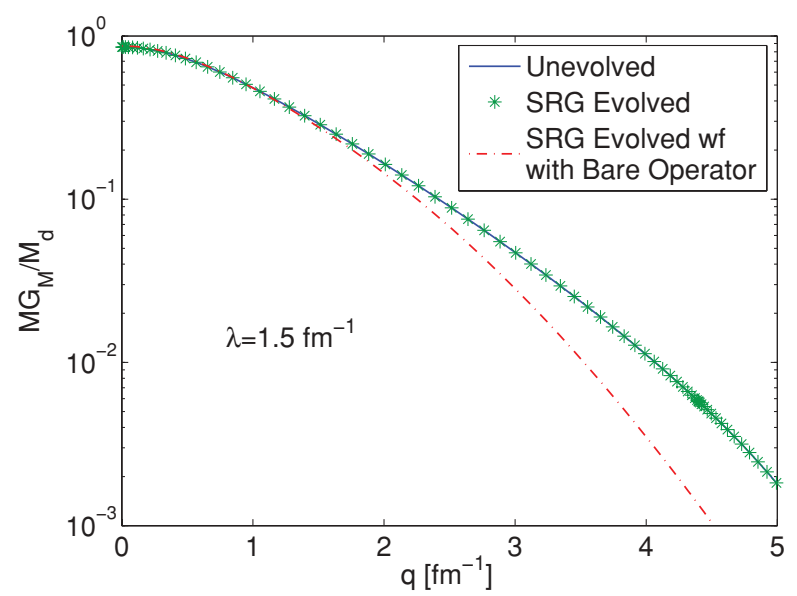

(c)

FIG. 11. (Color online) Deuteron form factors $G_{\mathrm{C}}, G_{\mathrm{Q}}$, and $G_{\mathrm{M}}$ using the isoscalar electric form factor parametrization from Ref. [24]. $M$ is the nucleon mass and $M_{d}$ is the mass of the deuteron. The wave function is derived from the NNLO 550/600-MeV potential and the evolution is run to $\lambda=1.5 \mathrm{fm}^{-1}[25]$.

alternative $\mathrm{RG}$ formulation) in the past to demonstrate a significantly improved convergence of the binding energies [26,27]. We choose to adapt a simple ansatz for the deuteron used in those calculations to make our point for the SRG. In particular, we take the (un-normalized) ${ }^{3} S_{1}$ and ${ }^{3} D_{1}$ partial waves to be

$$
\begin{aligned}
& u(k)=\frac{1}{\left(k^{2}+\gamma^{2}\right)\left(k^{2}+\mu^{2}\right)} e^{-\left(\frac{k^{2}}{\lambda^{2}}\right)^{2},} \\
& w(k)=\frac{a k^{2}}{\left(k^{2}+\gamma^{2}\right)\left(k^{2}+v^{2}\right)^{2}} e^{-\left(\frac{k^{2}}{\lambda^{2}}\right)^{2}},
\end{aligned}
$$

where $\gamma, \mu, v$, and $a$ are variational parameters. The exponential factors are chosen to match the asymptotic suppression of the wave function resulting from the decoupling of the interaction according to Eq. (12). The energy is minimized with respect to the variational parameters at various $\lambda$ for the three different potentials used in this article. The binding-energy results are shown in Fig. 14. Without evolution, the AV18 and $\mathrm{N}^{3} \mathrm{LO}$ trial wave functions are not even bound, and the NNLO wave function accounts for less than half the binding energy. With evolution, the AV18 and N3LO wave functions begin to bind at $\lambda \approx 7 \mathrm{fm}^{-1}$ and $\lambda \approx 4.5 \mathrm{fm}^{-1}$, respectively, and when the evolution is taken further, the trial wave function is able to reproduce the exact binding energies to within $\approx 1 \mathrm{keV}$.

Examining the matrix elements of operators which initially have strength concentrated over a range of different momenta-such as the occupation operators with respect to momenta $q$-provides a stricter test of the variational solution to the evolved wave functions and the sensitivity of evolved operators to them. The initial AV18 potential has particularly strong correlations at high momenta. If we look at the evolved occupation operators in Fig. 15, we see three curves: one with a wave function near the binding threshold, one at about half the binding energy, and one that is well converged with respect to the binding energy (evolved to $\lambda=6.0,4.0$, and $1.5 \mathrm{fm}^{-1}$, respectively). Near the binding threshold, the momentum distribution is reproduced rather poorly, but at smaller $\lambda$ the curve improves, and once the binding energies are converged the operator expectation values are also converged to approximately $1 \%$ or better.

The same pattern holds for other operators and interactions; that is, the operator matrix elements are not sensitive to the 


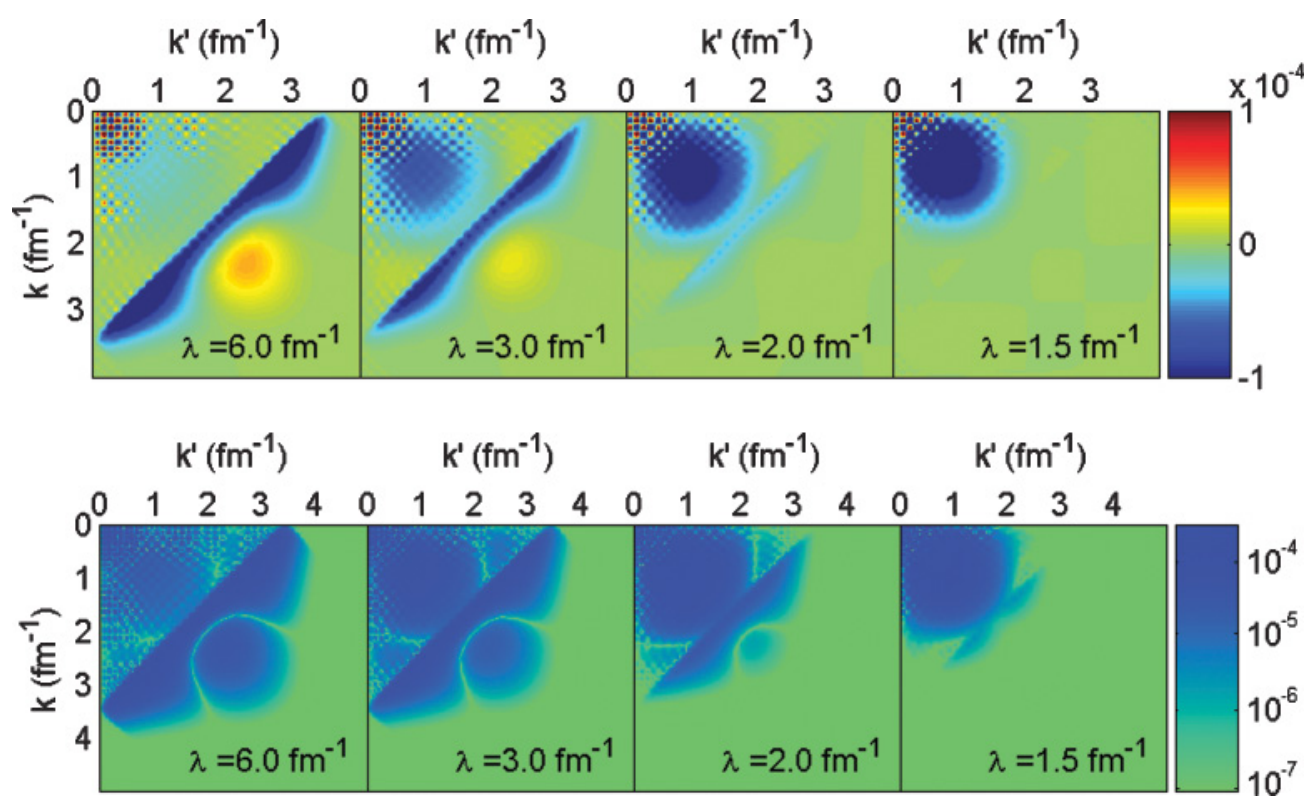

FIG. 12. (Color online) Integrand of $G_{\mathrm{M}}$ at $q=6.90 \mathrm{fm}^{-1}$ evolved from $\lambda=6 \mathrm{fm}^{-1}$ to $\lambda=1.5 \mathrm{fm}^{-1}$ using the NNLO 550/600-MeV potential with a linear scale (top) and a logarithmic scale of the magnitude (bottom).

fine details of the evolved wave function. The magnetic form factor of the deuteron using the NNLO potential, for example, is shown in Fig. 16. Not only does this operator pick up strength in both partial wave states of the deuteron, but also their coupling. Again, for a variational wave function at half binding energy $\left(\lambda=5.0 \mathrm{fm}^{-1}\right)$ the matrix elements deviate significantly from the direct, nonvariational calculation, but when the binding energy is converged (at $\lambda=1.2 \mathrm{fm}^{-1}$ ), the form factor expectation values are reproduced to better than $1 \%$.

\section{OPERATOR FACTORIZATION}

In this section, we consider in more detail the expectation value in a low-energy bound state of operators that initially have strength only at high momentum. The momentum distribution of the deuteron at large $q$ is our prototype. The momentum distribution for the initial potentials in Fig. 2 show structure at high momenta because of the short-range repulsion in the potential (particularly for AV18). When evolved to low momentum, this structure disappears and the deuteron wave function will select the low-momentum part

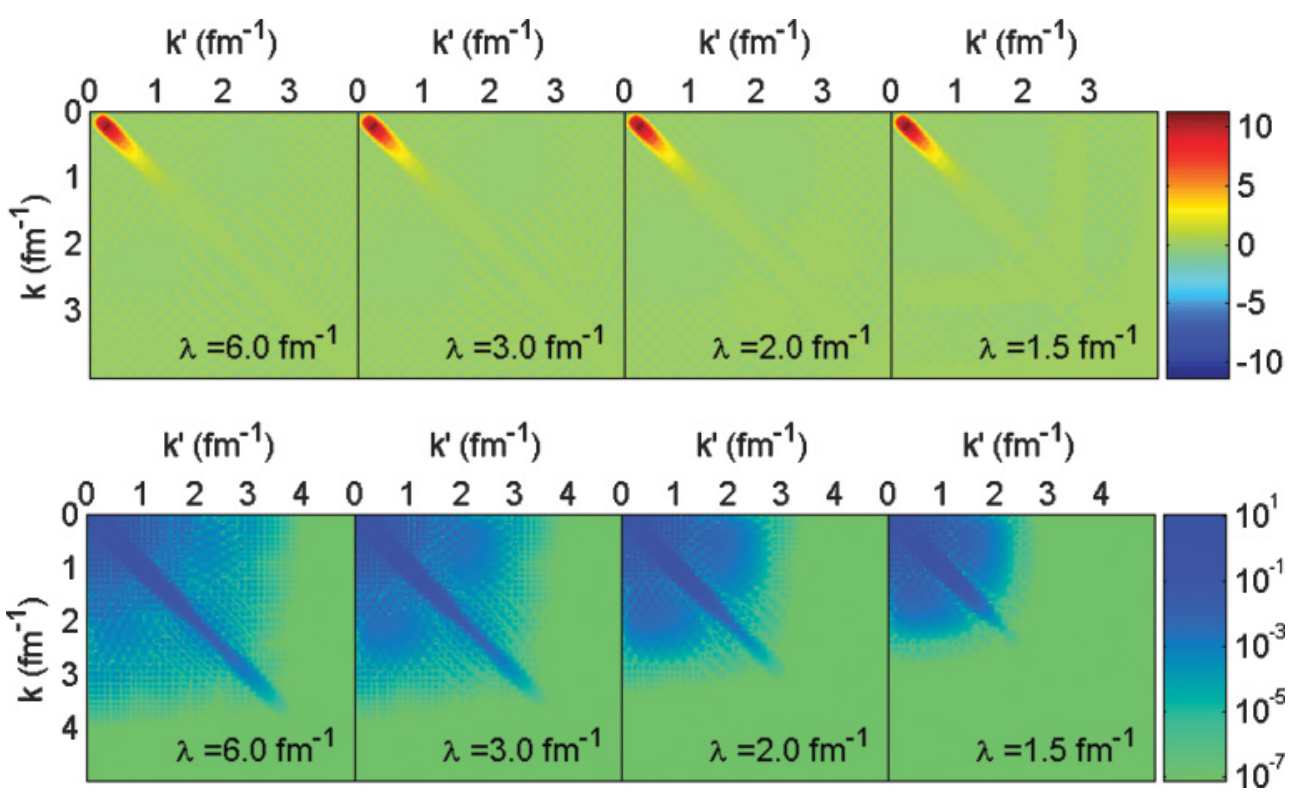

FIG. 13. (Color online) Integrand of $G_{\mathrm{M}}$ at $q=0.34 \mathrm{fm}^{-1}$ evolved from $\lambda=6 \mathrm{fm}^{-1}$ to $\lambda=1.5 \mathrm{fm}^{-1}$ using the NNLO $550 / 600-\mathrm{MeV}$ potential with a linear scale (top) and a logarithmic scale of the magnitude (bottom). 


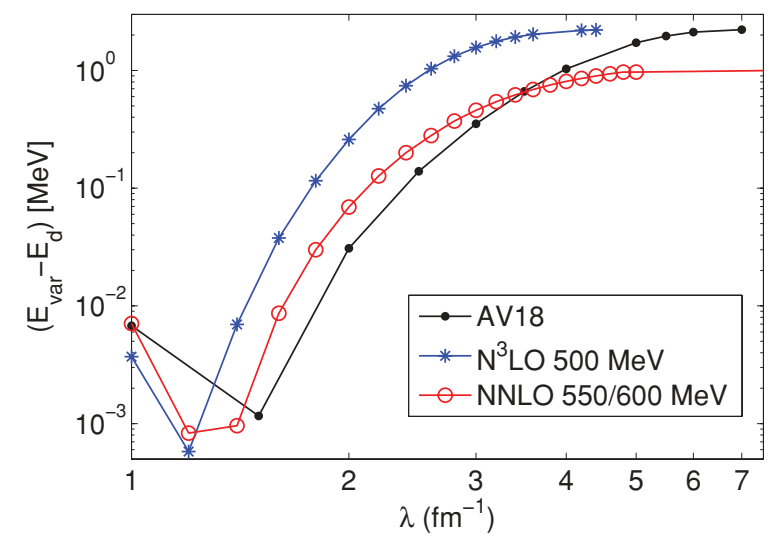

FIG. 14. (Color online) Deviation from $E_{d}$ of the best variational energy as a function of SRG decoupling parameter for the wave function ansatz of Eq. (31). $E_{d}$ is the deuteron-binding energy for each interaction derived via a full eigenvalue calculation of the Hamiltonian.

of the evolved operator. But this evolved operator must still reflect the external high-momentum scale. We can anticipate simplifications by exploiting this separation of scales provided by the SRG; in particular, we expect a factorization of the evolved operator based on operator-product-expansion (OPE) arguments applied to nonrelativistic effective theories [28,29].

\section{A. Numerical verification of factorization}

Previous calculations of the deuteron momentum distribution suggested that the unitary evolution operator, $U_{\lambda}(k, q){ }^{2}$ factorizes into a function of $k$ times a function of $q, U_{\lambda}(k, q) \rightarrow$ $K_{\lambda}(k) Q_{\lambda}(q)$, for $k<\lambda$ and $q \gg \lambda$ [13]. To numerically

\footnotetext{
${ }^{2}$ Because $\lambda$ is an important momentum scale in our factorization discussion, we use the notation $U_{\lambda}$ with $\lambda=s^{-1 / 4}$ rather than $U_{s}$ in this section.
}

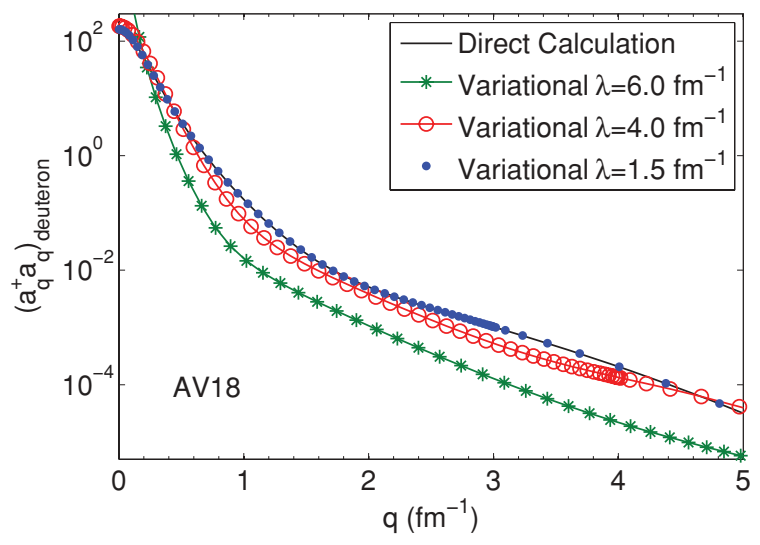

FIG. 15. (Color online) The momentum distribution in the deuteron as given by the expectation value of the evolved occupation operator $U a_{q}^{\dagger} a_{q} U^{\dagger}$ using the variational wave functions derived from the Salpeter ansatz and the AV18 potential evolved to $\lambda=6.0,4.0$, and $1.5 \mathrm{fm}^{-1}$. The direct calculation is from a full eigenvector solution of the Hamiltonian.

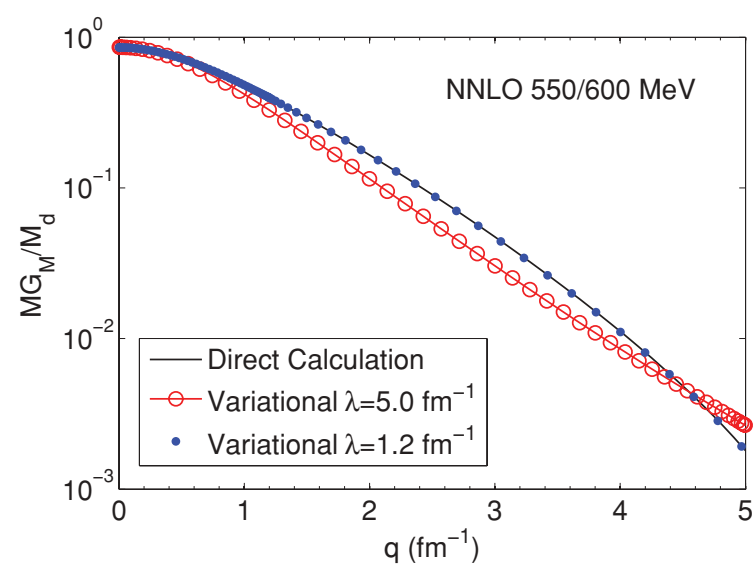

FIG. 16. (Color online) Deuteron form factor $G_{\mathrm{M}}$ using the isoscalar electric form factor parametrization from Ref. [24]. $M$ is the nucleon mass and $M_{d}$ is the mass of the deuteron. The variational wave functions are derived from the Salpeter ansatz and the NNLO $550 / 600-\mathrm{MeV}$ potential evolved to $\lambda=5.0 \mathrm{fm}^{-1}$ and $\lambda=1.2 \mathrm{fm}^{-1}$. The operators are evolved consistently. The direct calculation is from a full eigenvector solution of the Hamiltonian.

test for factorization in the unitary transformation, we use transformations generated via Eq. (15) and the evolution of $N N$ potentials, and consider the ratio

$$
\frac{U_{\lambda}\left(k_{i}, q\right)}{U_{\lambda}\left(k_{0}, q\right)} \stackrel{?}{\longrightarrow} \frac{K_{\lambda}\left(k_{i}\right) Q_{\lambda}(q)}{K_{\lambda}\left(k_{0}\right) Q_{\lambda}(q)},
$$

holding $k_{i}$ and $k_{0}$ constant with $k_{0} \ll \lambda$. If there is factorization, the $q$ dependence should cancel; that is, for $k\langle\lambda$ and $q \gg \lambda$, we should find

$$
\frac{U_{\lambda}\left(k_{i}, q\right)}{U_{\lambda}\left(k_{0}, q\right)} \approx \frac{K_{\lambda}\left(k_{i}\right)}{K_{\lambda}\left(k_{0}\right)} .
$$

In Figs. 17 and 18 we plot the ratio in Eq. (32) versus $q$ for representative cases. The signature of factorization is a plateau in $q$. The shaded regions are where $q \leqslant \lambda$. In all cases, there is no factorization in this region, consistent with the requirement that $q \gg \lambda$. In the unshaded region we see definite plateaus for $q>\lambda$ as long as $k_{i}<\lambda$, with diminishing prominence as $k_{i}$ increases (they disappear for $k_{i}>\lambda$ ). Thus, we have at least a qualitative verification of factorization. Note that Fig. 18(b) shows that for larger $\lambda$ the clean factorization breaks down (as well as restricting the applicable domain).

The singular value decomposition (SVD) can be used as a tool to quantitatively analyze the extent to which $U_{\lambda}$ factorizes. The SVD of a matrix $M$ can be expressed in general as an outer product expansion,

$$
M=\sum_{i=1}^{r} d_{i} \mathbf{u}_{i} \mathbf{v}_{i}^{t},
$$

where $r$ is the rank of the matrix and the $d_{i}$ are the singular values (in order of decreasing value). The idea is that if the first singular value, $d_{1}$, is sufficiently large compared to the others, the first term dominates and we have a factorized approximation. We can apply this to $U_{\lambda}$ in the region where high and low momentum couple. Thus, the vector $\mathbf{u}_{1}$ would correspond to the low-momentum function $K_{\lambda}(k)$ from Eq. (32) and $\mathbf{v}_{1}$ 


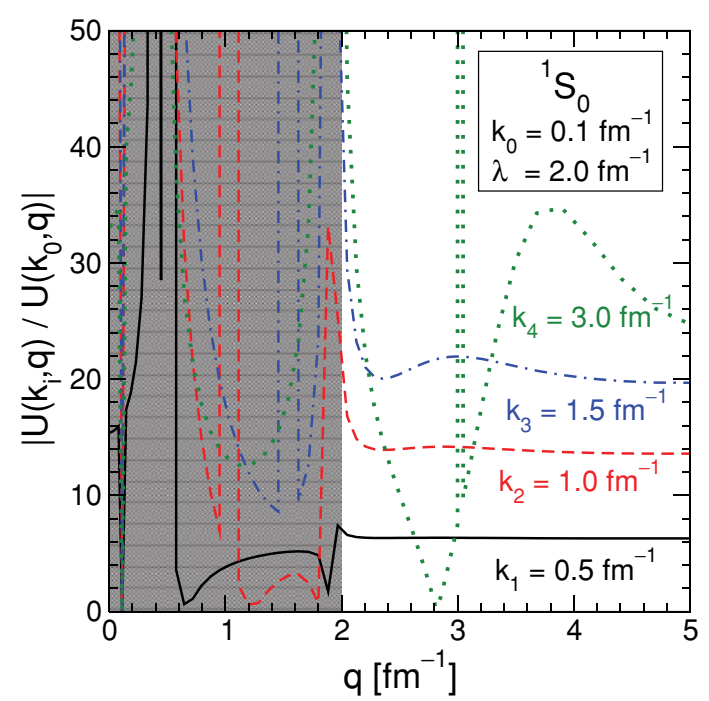

(a)

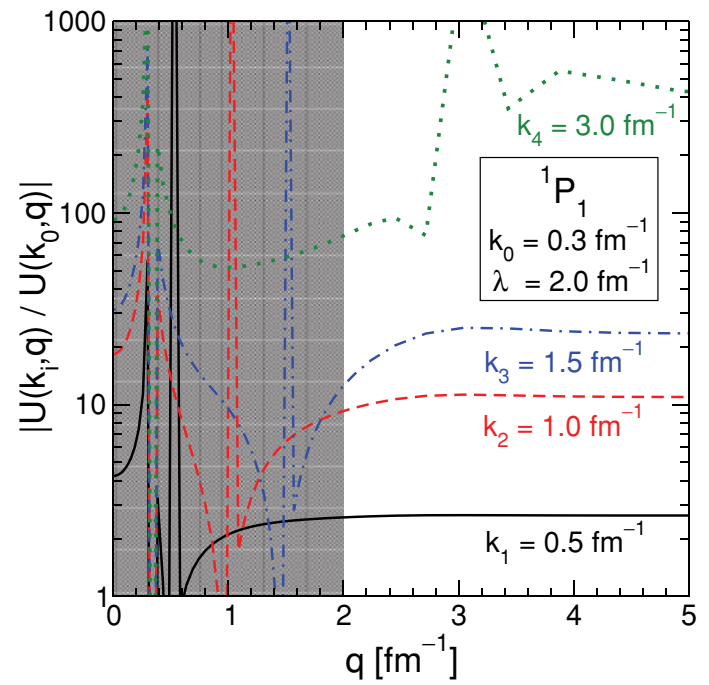

(b)

FIG. 17. (Color online) Numerical tests of factorization of the unitary transformation $U_{\lambda}(k, q)$ by plotting the ratio in Eqs. (32) and (33) as a function of $q$ for fixed $k_{0}$ and several values of $k_{i}$. Plateaus in $q$ indicate factorization. The unitary transformations are generated from the Argonne $v_{18}$ (AV18) [16] potential evolved to $\lambda=2 \mathrm{fm}^{-1}$ in the (a) ${ }^{1} S_{0}$ and (b) ${ }^{1} P_{1}$ partial waves. The shaded region marks $q<\lambda$.

to $Q_{\lambda}(q)$. If valid, one can calculate the factorized operator using the unitary transformation obtained directly from the SVD. Moreover, the expansion provided by the SVD allows us to make systematic corrections to the factorized unitary transformation and the operators evolved with it.

To test if such an expansion can be used, the first few singular values have been calculated in Table II for the $q>\lambda$ and $k<\lambda$ region of the SRG unitary transformations for several different potentials, each evolved to $\lambda=2 \mathrm{fm}^{-1}$. That is, the SVD is applied to the matrix obtained when elements of $U_{\lambda}(k, q)$ with $k>\Lambda$ and $q<\Lambda$ are set to zero; in practice a cutoff $\Lambda \approx 2.5 \mathrm{fm}^{-1}$ is used to ensure that we are in the region where off-diagonal coupling is strongly suppressed everywhere in the Hamiltonian. The dominance of $d_{1}$ in each case is promising.

To test if a truncated outer product sum is a good approximation to the contribution from $k\langle\Lambda, q\rangle \Lambda$, we consider the errors in some representative expectation values in Table III for several levels of truncation. The "zeroth-order" contribution is from the matrix where $k>\Lambda$ and $q>\Lambda$ is set to zero (this is denoted by SVD $=0$ in the table). The first-order (SVD $=1$ in the table) contribution uses the same matrix plus the approximation of $U_{\lambda}(k, q)$ for $k<\Lambda$ and $q>\Lambda$ given by the first outer product in the SVD expansion. The second-order approximation uses two outer products, etc. The occupation and charge form factor operators shown here are chosen to

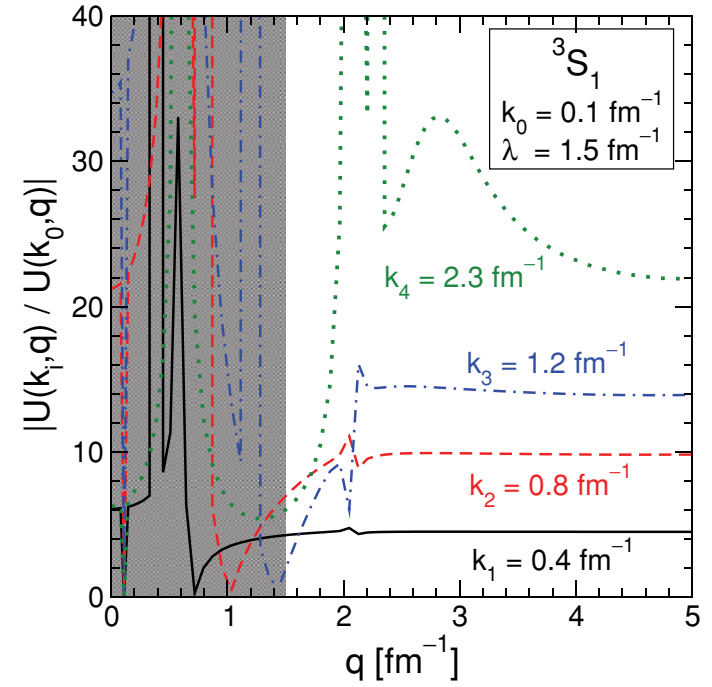

(a)

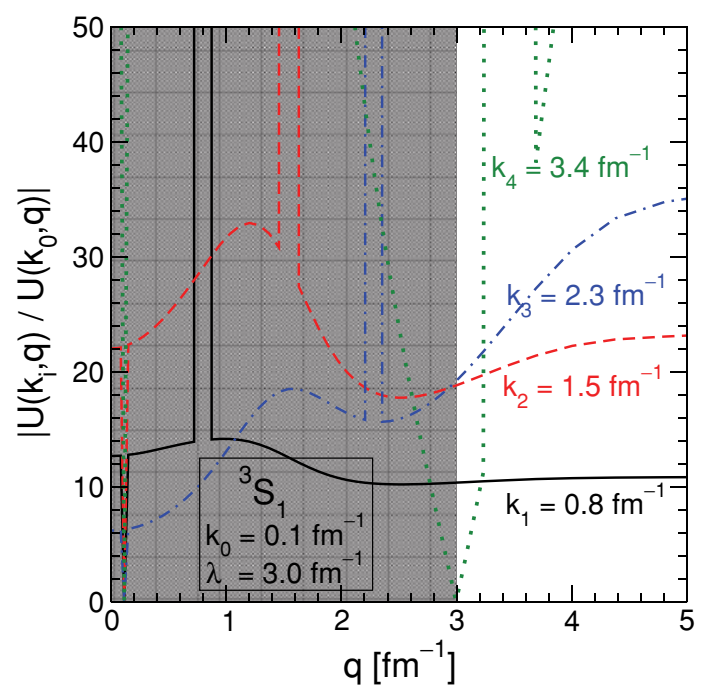

(b)

FIG. 18. (Color online) Same as Fig. 17 but for the ${ }^{3} S_{1}$ partial wave and $\lambda$ of (a) $1.5 \mathrm{fm}^{-1}$ and (b) $3 \mathrm{fm}^{-1}$. 
TABLE II. Singular values of the unitary transformation $U(k, q)$ for $q>\Lambda$ and $k<\Lambda$ (see discussion in text; units in $\mathrm{fm}^{-1}$ ) corresponding to the given potentials at $\lambda=2 \mathrm{fm}^{-1}$ in the ${ }^{1} S_{0}$ partial wave and ${ }^{3} S_{1-}{ }^{3} D_{1}$ coupled channel.

\begin{tabular}{lcccccccc}
\hline \hline Potential & \multicolumn{3}{c}{${ }^{1} S_{0}$} & & \multicolumn{3}{c}{${ }^{3} S_{1}-{ }^{3} D_{1}$} \\
\cline { 2 - 4 } \cline { 7 - 9 } & $d_{1}$ & $d_{2}$ & $d_{3}$ & & $d_{1}$ & $d_{2}$ & $d_{3}$ \\
\hline AV18 & 0.763 & 0.033 & 0.007 & & 0.671 & 0.015 & 0.008 \\
N3LO 500 MeV & 1.423 & 0.221 & 0.015 & & 1.873 & 0.225 & 0.044 \\
N3LO 550/600 MeV & 3.074 & 0.380 & 0.061 & & 4.195 & 0.587 & 0.089 \\
\hline \hline
\end{tabular}

illustrate the effects of the factorized approximation at various momenta. Additionally, the initial occupation operator has no off-diagonal strength, whereas the initial charge form-factor operators have relatively substantial off-diagonal contributions at large values of $q$; this is significant for the applicability of factorization to an operator, as we see below. So, what we find at low momentum for the occupation operator is that it is essentially exact, up to errors resulting from decoupling and truncation of the wave function, and it is the same with or without the SVD approximation. This error increases, as expected, for larger $\lambda$. Because $G_{C}$ is more diffuse initially, we see a small improvement even at small momenta when using the SVD approximations.

For the occupation operator at high momenta (well above the cutoff), the error is $100 \%$ without an approximation to $U_{\lambda}(k, q)$ because there is a hard cutoff and the initial operator is localized in the upper region of momentum space. However, with just one term in the SVD expansion we recover that expectation value for $\lambda=1.5 \mathrm{fm}^{-1}$ to better than $1 \%$, and the situation improves with additional terms in the expansion. At $\lambda=3.0 \mathrm{fm}^{-1}$ decoupling is evidently not sufficient for this approximation to work well. At very large values of momenta (e.g., $q \approx 6.9 \mathrm{fm}^{-1}$ ) the charge form factor shows improvement with the SVD approximation, but because this operator has significant off-diagonal strength, the improvement is not as pronounced. At a value of $q \approx 3.0 \mathrm{fm}^{-1}$ it is evident that the SVD still improves the relative error. However, recall that the strength in the form factors is larger around $\approx \frac{1}{2} q$.

\section{B. Connection to the operator product expansion}

The OPE was developed for the evaluation of singular products of local field operators at small separation. In our case, where such operators are treated as matrices and we typically work in momentum representation, the focus becomes low-momentum matrix elements of a product in which high-momentum states dominate the intermediate sum. This leads us directly to consider low- to high-momentum matrix elements of SRG-evolved operators, and a generic analysis is then based on the study of $U_{\lambda}(k, q)$ for $k\langle\lambda$ and $q \gg \lambda$.

The utility of the OPE rests on factorization; short-distance details decouple from long-distance dynamics. Factorization enables one, for example, to separate the momentum and distance scales in hard-scattering processes in terms of perturbative QCD and parton distribution functions. In our case, factorization is the direct result of decoupling. It provides tools that let us parametrize the high-momentum components of operators that would normally require degrees of freedom we do not retain. We can, for example, build effective few-body operators containing state-independent functions of high momenta that can be measured directly in few-body experiments. These operators can then be employed to make predictions for $A$-body systems.

Consider a generic operator, $\widehat{O}_{\lambda}=U_{\lambda} \widehat{O} U_{\lambda}^{\dagger}$, and employ the spectral representation for $U_{\lambda}$ :

$$
U_{\lambda}(k, q)=\sum_{\alpha}\left\langle k \mid \psi_{\alpha}(\lambda)\right\rangle\left\langle\psi_{\alpha}(\infty) \mid q\right\rangle .
$$

The OPE deals with cases in which the unevolved operator is dominated by high momenta (e.g., $a_{q}^{\dagger} a_{q}$ with large $q$ is the simplest paradigm), and we focus on $k<\lambda$ and $q \gg \lambda$. For $k<\lambda$ we exploit the fact that low-momentum components of high-energy eigenstates of $H_{\lambda}$ are exponentially suppressed because of decoupling. As a result, the sum is dominated by low-energy states,

$$
U_{\lambda}(k, q) \approx \sum_{E_{\alpha} \ll \lambda^{2}}\left\langle k \mid \psi_{\alpha}(\lambda)\right\rangle\left\langle\psi_{\alpha}(\infty) \mid q\right\rangle .
$$

Once the sum is restricted, we can turn our focus to approximating the high-momentum components of the unevolved low-energy states. This analysis is closely related to Lepage's discussion of the OPE analysis of wave functions, which leads him to write in position representation [28]:

$$
\begin{aligned}
\Psi_{\text {true }}(r)= & \bar{\gamma}(r) \int d^{3} r \Psi_{\mathrm{eff}} \delta_{a}^{3}(\boldsymbol{r})+\bar{\eta}(r) a^{2} \\
& \times \int d^{3} r \Psi_{\mathrm{eff}} \nabla^{2} \delta_{a}^{3}(\boldsymbol{r})+\mathcal{O}\left(a^{4}\right),
\end{aligned}
$$

\begin{tabular}{|c|c|c|c|c|c|c|c|c|}
\hline \multirow[t]{2}{*}{ Operator } & \multicolumn{4}{|c|}{$\mathrm{SRG} \lambda=1.5 \mathrm{fm}^{-1}$} & \multicolumn{4}{|c|}{$\operatorname{SRG} \lambda=3.0 \mathrm{fm}^{-1}$} \\
\hline & SVD & $q=0.34$ & $q=3.01$ & $q=6.90$ & SVD & $q=0.34$ & $q=3.01$ & $q=6.90$ \\
\hline$\left\langle a_{q}^{\dagger} a_{q}\right\rangle$ & 0 & $7.61 \times 10^{-7}$ & 1.00 & & 0 & $1.06 \times 10^{-3}$ & 1.00 & \\
\hline With $\mathrm{N}^{3} \mathrm{LO}$ & $\begin{array}{l}1 \\
2\end{array}$ & $\begin{array}{l}7.61 \times 10^{-7} \\
7.61 \times 10^{-7}\end{array}$ & $\begin{array}{l}4.28 \times 10^{-3} \\
4.79 \times 10^{-4}\end{array}$ & & $\begin{array}{l}1 \\
2\end{array}$ & $\begin{array}{l}1.06 \times 10^{-3} \\
1.06 \times 10^{-3}\end{array}$ & $\begin{array}{l}6.36 \times 10^{-1} \\
6.35 \times 10^{-1}\end{array}$ & \\
\hline $\begin{array}{l}G_{\mathrm{C}}(q) \\
\text { With NNLO }\end{array}$ & $\begin{array}{l}0 \\
1 \\
2\end{array}$ & $\begin{array}{l}6.90 \times 10^{-4} \\
1.28 \times 10^{-7} \\
1.04 \times 10^{-6}\end{array}$ & $\begin{array}{l}5.01 \times 10^{-3} \\
8.90 \times 10^{-5} \\
2.10 \times 10^{-5}\end{array}$ & $\begin{array}{l}8.93 \times 10^{-1} \\
4.06 \times 10^{-2} \\
4.18 \times 10^{-2}\end{array}$ & $\begin{array}{l}0 \\
1 \\
2\end{array}$ & $\begin{array}{l}4.10 \times 10^{-4} \\
1.63 \times 10^{-4} \\
1.63 \times 10^{-4}\end{array}$ & $\begin{array}{l}3.36 \times 10^{-3} \\
2.66 \times 10^{-4} \\
3.04 \times 10^{-4}\end{array}$ & $\begin{array}{l}8.92 \times 10^{-1} \\
4.00 \times 10^{-1} \\
4.09 \times 10^{-1}\end{array}$ \\
\hline
\end{tabular}

TABLE III. Relative error of evolved operator matrix elements calculated using the SVD to factorize $U_{\lambda}(k, q)$ in the region where $k<\Lambda$ and $q>\Lambda$ (see discussion in text; units in $\mathrm{fm}^{-1}$ ). 
where the coefficient functions $\bar{\gamma}(r)$ and $\bar{\eta}(r)$ are stateindependent parametrizations of the short-distance physics, and $a$ is approximately the distance of the ultraviolet cutoff. In this section, we outline how SRG factorization can be understood more generally (and analytically) in the context of the OPE for nonrelativistic Schrödinger problems by deriving an analogous equation in momentum space for the SRG-evolved wave function.

To do so, we first define the projection operators

$$
\mathcal{P}_{\Lambda}=\int_{0}^{\Lambda} d \tilde{p}|p\rangle\langle p|
$$

and

$$
\mathcal{Q}_{\Lambda}=\int_{\Lambda}^{\infty} d \tilde{q}|q\rangle\langle q|
$$

where $\Lambda$ divides momentum space and $d \tilde{p} \equiv \frac{2}{\pi} p^{2} d p$ in the partial-wave momentum basis. This $\Lambda$ is to be distinguished from $\lambda$, which is the SRG evolution parameter and an approximate measure of decoupling in the evolved potential. We use $\psi_{\alpha}^{\lambda}$ to denote the eigenstates of the Hamiltonian ordered according to increasing energy $E_{\alpha}$ and evolved to $\lambda$ via the SRG. $H_{\lambda}$ and $V_{\lambda}$ represent the corresponding SRG-evolved Hamiltonian and potential. The initial, unevolved operators correspond to $\lambda=\infty$.

From the unevolved Schrödinger equation

$$
H_{\infty}\left|\psi_{\alpha}^{\infty}\right\rangle=E_{\alpha}\left|\psi_{\alpha}^{\infty}\right\rangle
$$

we can write

$$
\left(\begin{array}{cc}
\mathcal{P}_{\Lambda} H_{\infty} \mathcal{P}_{\Lambda} & \mathcal{P}_{\Lambda} H_{\infty} \mathcal{Q}_{\Lambda} \\
\mathcal{Q}_{\Lambda} H_{\infty} \mathcal{P}_{\Lambda} & \mathcal{Q}_{\Lambda} H_{\infty} \mathcal{Q}_{\Lambda}
\end{array}\right)\left(\begin{array}{c}
\mathcal{P}_{\Lambda} \psi_{\alpha}^{\infty} \\
\mathcal{Q}_{\Lambda} \psi_{\alpha}^{\infty}
\end{array}\right)=E_{\alpha}\left(\begin{array}{c}
\mathcal{P}_{\Lambda} \psi_{\alpha}^{\infty} \\
\mathcal{Q}_{\Lambda} \psi_{\alpha}^{\infty}
\end{array}\right)
$$

and thus for the " $\mathcal{Q}$ " space we have

$$
\begin{aligned}
\mathcal{Q}_{\Lambda}\left|\psi_{\alpha}^{\infty}\right\rangle & =\left(E_{\alpha}-\mathcal{Q}_{\Lambda} H_{\infty} \mathcal{Q}_{\Lambda}\right)^{-1} \mathcal{Q}_{\Lambda} H_{\infty} \mathcal{P}_{\Lambda} \mathcal{P}_{\Lambda}\left|\psi_{\alpha}^{\infty}\right\rangle \\
& =\left(E_{\alpha}-\mathcal{Q}_{\Lambda} H_{\infty} \mathcal{Q}_{\Lambda}\right)^{-1} \mathcal{Q}_{\Lambda} V_{\infty} \mathcal{P}_{\Lambda}\left|\psi_{\alpha}^{\infty}\right\rangle,
\end{aligned}
$$

where we have used $\left(\mathcal{P}_{\Lambda}\right)^{2}=\mathcal{P}_{\Lambda}, H_{\infty}=T+V_{\infty}$, and $\mathcal{Q}_{\Lambda} T \mathcal{P}_{\Lambda}=0$. For low-energy states $\psi_{\alpha}^{\infty}$ such that $\left|E_{\alpha}\right| \ll$ $\min \left[\left|E_{\mathcal{Q} H \mathcal{Q}}\right|\right]$ (where $E_{\mathcal{Q} H \mathcal{Q}}$ are the eigenvalues of $\mathcal{Q} H \mathcal{Q}$ ), we can neglect the $E_{\alpha}$ dependence. Also, assuming that the potential $V_{\infty}\left(q^{\prime}, p\right)$ is slowly varying with respect to $p$ compared to $\psi_{\alpha}^{\infty}(p)$ in the region $p<\Lambda$ and $q^{\prime} \gg \Lambda$, we can use the expansion

$$
\begin{aligned}
& \int_{0}^{\Lambda} d \tilde{p} V_{\infty}\left(q^{\prime}, p\right) \psi_{\alpha}^{\infty}(p) \\
& \left.\approx V_{\infty}\left(q^{\prime}, p^{\prime}\right)\right|_{p^{\prime}=0} \int_{0}^{\Lambda} d \tilde{p} \psi_{\alpha}^{\infty}(p) \\
& \quad+\left.\frac{d^{2}}{d p^{\prime 2}} V_{\infty}\left(q^{\prime}, p^{\prime}\right)\right|_{p^{\prime}=0} \int_{0}^{\Lambda} d \tilde{p} p^{2} \psi_{\alpha}^{\infty}(p)+\cdots
\end{aligned}
$$

to first order, combined with the fact that the low-energy states will have momentum components peaked at small $p$, to write

$$
\begin{aligned}
\left\langle q \mid \psi_{\alpha}^{\infty}\right\rangle \approx & -\int_{\Lambda}^{\infty} d \tilde{q}^{\prime} \int_{0}^{\Lambda} d \tilde{p}\langle q| \frac{1}{\mathcal{Q}_{\Lambda} H_{\infty} \mathcal{Q}_{\Lambda}} \\
& \times\left|q^{\prime}\right\rangle V_{\infty}\left(q^{\prime}, 0\right) \psi_{\alpha}^{\infty}(p) .
\end{aligned}
$$

Tests indicate that these assumptions are valid for realistic $N N$ potentials.

Further, we see empirically via Fig. 2 that $\mathcal{P}_{\Lambda}\left|\psi_{\alpha}^{\infty}\right\rangle \approx$ $Z(\lambda)\left|\psi_{\alpha}^{\lambda}\right\rangle$ when $\lambda \gtrsim \Lambda$ (this is consistent with our understanding that the SRG with $G_{s}=T_{\text {rel }}$ renormalizes/suppresses only the short-distance components of the wave function for values of $\lambda$ considered here). Thus, setting $\Lambda=\lambda$ and defining

$$
\gamma^{\lambda}(q) \equiv-\int_{\lambda}^{\infty} d \tilde{q}^{\prime}\left\langle q\left|\frac{1}{\mathcal{Q}_{\lambda} H_{\infty} \mathcal{Q}_{\lambda}}\right| q^{\prime}\right\rangle V_{\infty}\left(q^{\prime}, 0\right)
$$

we have

$$
\psi_{\alpha}^{\infty}(q) \approx \gamma^{\lambda}(q) \int_{0}^{\lambda} d \tilde{p} Z(\lambda) \psi_{\alpha}^{\lambda}(p) .
$$

So we see that the high-momentum components of low-energy eigenstates can be factorized into a state-independent function $\gamma^{\lambda}(q)$, which summarizes the short-distance behavior of the wave function, and a coefficient (given by an integral over the renormalized wave function) that gives the contribution from the long-distance structure of the state. Moreover, if we include higher-order corrections resulting from the expansion of $\int_{0}^{\lambda} d \tilde{p} V_{\infty}\left(q^{\prime}, p\right) \psi_{\alpha}^{\infty}(p)$ about $p=0$, we recover the analog to Lepage's OPE [Eq. (37)] in momentum space for the shortdistance structure of a wave function. It is given by

$$
\begin{aligned}
\psi_{\alpha}^{\infty}(q) \approx & \gamma^{\lambda}(q) \int_{0}^{\lambda} d \tilde{p} Z(\lambda) \psi_{\alpha}^{\lambda}(p) \\
& +\eta^{\lambda}(q) \int_{0}^{\lambda} d \tilde{p} p^{2} Z(\lambda) \psi_{\alpha}^{\lambda}(p)+\cdots,
\end{aligned}
$$

where $\gamma^{\lambda}(q)$ is given previously and

$$
\eta^{\lambda}(q) \equiv-\left.\int_{\lambda}^{\infty} d \tilde{q}^{\prime}\left\langle q\left|\frac{1}{\mathcal{Q}_{\lambda} H_{\infty} \mathcal{Q}_{\lambda}}\right| q^{\prime}\right\rangle \frac{\partial^{2}}{\partial p^{2}} V_{\infty}\left(q^{\prime}, p\right)\right|_{p=0} .
$$

Now, from the definition of the SRG unitary evolution operator in Eq. (15), in the region $k<\lambda$ and $q \gg \lambda$ we can use the leading-order term of our OPE to write

$$
\begin{aligned}
U_{\lambda}(k, q) & =\sum_{\alpha}^{\infty}\left\langle k \mid \psi_{\alpha}^{\lambda}\right\rangle\left\langle\psi_{\alpha}^{\infty} \mid q\right\rangle \\
& \approx\left[\sum_{\alpha}^{\left|E_{\alpha}\right| \ll\left|E_{\mathcal{Q} H \mathcal{Q}}\right|}\left\langle k \mid \psi_{\alpha}^{\lambda}\right\rangle \int_{0}^{\lambda} d \tilde{p} Z(\lambda) \psi_{\alpha}^{\lambda \dagger}(p)\right] \gamma^{\lambda}(q) \\
& \equiv K_{\lambda}(k) Q_{\lambda}(q)
\end{aligned}
$$

where the sum is only over states in the " $\mathcal{P}$ " space thanks to decoupling. Thus, we can understand the factorization of $U_{\lambda}$ as a general consequence of our ability to factorize the high-momentum components of low-energy nuclear wave functions via an OPE plus decoupling in the SRG-evolved Hamiltonian. Moreover, because $\psi_{\alpha}^{\lambda}(k)$ to good approximation has no support when $k<\lambda$ for $\alpha$ in the " $\mathcal{Q}$ " space, we can extend the $\alpha$ sum in Eq. (49) to the full space and apply closure to find

$$
\begin{aligned}
U_{\lambda}(k, q) & \approx\left[Z(\lambda) \int_{0}^{\lambda} d \tilde{p} \sum_{\alpha}^{\infty}\left\langle k \mid \psi_{\alpha}^{\lambda}\right\rangle\left\langle\psi_{\alpha}^{\lambda} \mid p\right\rangle\right] \gamma^{\lambda}(q) \\
& \approx Z(\lambda) \gamma^{\lambda}(q) .
\end{aligned}
$$


Thus, to a first approximation, $K_{\lambda}(k)$ is a constant factor.

To gain insight into the implications of this factorization, we consider the expectation value of $a_{q}^{\dagger} a_{q}$ in a low-energy state, the deuteron. Because we know that strength in the evolved number operator expectation value decouples from high-momentum contributions in the deuteron, we can write

$$
\begin{aligned}
& \left\langle\psi_{d}^{\lambda}\left|\left(a_{q}^{\dagger} a_{q}\right)_{\lambda}\right| \psi_{d}^{\lambda}\right\rangle \\
& =\left\langle\psi_{d}^{\lambda}\left|U_{\lambda}\left(a_{q}^{\dagger} a_{q}\right) U_{\lambda}^{\dagger}\right| \psi_{d}^{\lambda}\right\rangle \approx \int_{0}^{\lambda} d \tilde{k}^{\prime} \int_{0}^{\infty} d \tilde{q}^{\prime} \int_{0}^{\infty} d \tilde{q}^{\prime \prime} \\
& \quad \times \int_{0}^{\lambda} d \tilde{k} \psi_{d}^{\lambda \dagger}\left(k^{\prime}\right) U_{\lambda}\left(k^{\prime}, q^{\prime}\right) \delta\left(q^{\prime}-q\right) \delta\left(q^{\prime \prime}-q^{\prime}\right) \\
& \quad \times U_{\lambda}\left(q^{\prime \prime}, k\right) \psi_{d}^{\lambda}(k) \\
& =\int_{0}^{\lambda} d \tilde{k}^{\prime} \int_{0}^{\lambda} d \tilde{k} \psi_{d}^{\lambda \dagger}\left(k^{\prime}\right) U_{\lambda}\left(k^{\prime}, q\right) U_{\lambda}(q, k) \psi_{d}^{\lambda}(k) .
\end{aligned}
$$

For a low-momentum operator, one with $q<\lambda$, the expectation value thus depends only on the low-momentum details of the wave function (original and evolved). For $q \gg \lambda$, however, we can make use of factorization and set $U(k, q) \rightarrow$ $K_{\lambda}(k) Q_{\lambda}(q)$ to write

$$
\int_{0}^{\lambda} d \tilde{k}^{\prime} \int_{0}^{\lambda} d \tilde{k} \psi_{d}^{\lambda \dagger}\left(k^{\prime}\right) K_{\lambda}\left(k^{\prime}\right)\left[Q_{\lambda}(q) Q_{\lambda}(q)\right] K_{\lambda}(k) \psi_{d}^{\lambda}(k)
$$

from Eq. (51). Here we see that the expectation value of a high-momentum number operator is independent of the longdistance structure of the wave function. This is consistent with earlier calculations of the deuteron momentum distribution [13]. Again, as with decoupling in the potential, we appear to have a means by which long- and short-distance details can be separated for an operator evolved via the SRG.

The generalization of this result is straightforward. Consider the expectation value of an arbitrary operator, $O\left(q^{\prime}, q\right)$, in a low-energy state, $\psi_{\text {low }}^{\lambda}$. Because decoupling is valid for operator expectation values in a momentum basis (as we have seen via the expectation value integrand plots in Secs. II and III), we can write

$$
\begin{aligned}
& \left\langle\psi_{\text {low }}^{\lambda}\left|U_{\lambda} \widehat{O} U_{\lambda}^{\dagger}\right| \psi_{\text {low }}^{\lambda}\right\rangle \\
& \approx \int_{0}^{\lambda} d \tilde{k}^{\prime} \int_{0}^{\infty} d \tilde{q}^{\prime} \int_{0}^{\infty} d \tilde{q} \int_{0}^{\lambda} d \tilde{k} \\
& \quad \times\left[\psi_{\text {low }}^{\lambda}\left(k^{\prime}\right)\right]^{\dagger} U_{\lambda}\left(k^{\prime}, q^{\prime}\right) O\left(q^{\prime}, q\right) U_{\lambda}(q, k) \psi_{\text {low }}^{\lambda}(k) .
\end{aligned}
$$

We separate the integrals over the operator in the expectation value and apply factorization to set $U(k, q) \rightarrow K_{\lambda}(k) Q_{\lambda}(q)$ in the region where $k\langle\lambda$ and $q \gg \lambda$. If the unevolved operator has coupling between high and low momentum above the the factorization cut, then there is no great simplification. However, if the unevolved operator does not have coupling of high and low momentum above the factorization cut, factorization will allow us to separate out the high- and lowmomentum structure of an operator into two contributions:

$$
\begin{aligned}
& \int_{0}^{\lambda} d \tilde{k}^{\prime} \int_{0}^{\lambda} d \tilde{q}^{\prime} \int_{0}^{\lambda} d \tilde{q} \int_{0}^{\lambda} d \tilde{k} \\
& \quad \times\left[\psi_{\text {low }}^{\lambda}\left(k^{\prime}\right)\right]^{\dagger} U_{\lambda}\left(k^{\prime}, q^{\prime}\right) O\left(q^{\prime}, q\right) U_{\lambda}(q, k) \psi_{\text {low }}^{\lambda}(k)
\end{aligned}
$$

and

$$
\begin{aligned}
& \int_{0}^{\lambda} d \tilde{k}^{\prime} \int_{\lambda}^{\infty} d \tilde{q}^{\prime} \int_{\lambda}^{\infty} d \tilde{q} \int_{0}^{\lambda} d \tilde{k}\left[\psi_{\text {low }}^{\lambda}\left(k^{\prime}\right)\right]^{\dagger} K_{\lambda}\left(k^{\prime}\right) \\
& \quad \times\left[Q_{\lambda}\left(q^{\prime}\right) O\left(q^{\prime}, q\right) Q_{\lambda}(q)\right] K_{\lambda}(k) \psi_{\text {low }}^{\lambda}(k) .
\end{aligned}
$$

This is analogous to what was found for the number operator.

Thus, we see that the breakdown of contributions to the expectation value of a general operator is consistent with our interpretation of the SRG flow equations as a means by which one can achieve a separation of scales in the evaluation of nuclear few- and many-body problems. We see explicitly here that the effects of a low-momentum probe of the ground-state wave function depends (almost entirely) on the low-momentum details of the renormalized wave function. Likewise, the effect of a high-momentum probe is largely independent of the low-momentum structure. It is only for operators which probe the coupling of high and low momentum (long and short distance) details of the wave function that we must consider the full momentum-space evolution of the operator. For the operators that have been considered in this paper, the latter is only true of the electromagnetic form factors at relatively high momenta (beyond the typical regime of interest for nuclear structure); for any operators which weakly couple high and low momentum, these terms can be neglected.

To summarize, we can write the expectation value of an operator that has weak coupling between high and low momentum as

$$
\begin{aligned}
& \left\langle\psi_{\text {low }}^{\lambda}\left|U_{\lambda} \widehat{O} U_{\lambda}^{\dagger}\right| \psi_{\text {low }}^{\lambda}\right\rangle \\
& \approx \int_{0}^{\lambda} d \tilde{k}^{\prime} \int_{0}^{\lambda} d \tilde{k}\left[\psi_{\text {low }}^{\lambda}\left(k^{\prime}\right)\right]^{\dagger}\left[\int_{0}^{\lambda} d \tilde{q}^{\prime} \int_{0}^{\lambda} d \tilde{q}\right. \\
& \quad \times U_{\lambda}\left(k^{\prime}, q^{\prime}\right) O\left(q^{\prime}, q\right) U_{\lambda}(q, k) \\
& \left.\quad+I_{Q O Q} K_{\lambda}\left(k^{\prime}\right) K_{\lambda}(k)\right] \psi_{\text {low }}^{\lambda}(k),
\end{aligned}
$$

where

$$
I_{Q O Q}=\int_{\lambda}^{\infty} d \tilde{q}^{\prime} \int_{\lambda}^{\infty} d \tilde{q} Q_{\lambda}\left(q^{\prime}\right) O\left(q^{\prime}, q\right) Q_{\lambda}(q) .
$$

By using factorization, we have seen that the expectation value breaks into a sum of two components: one which describes low-momentum structure, and a high-momentum component that factorizes into a piece depending on the low-momentum structure and another piece that is a universal function of high momentum $q$.

\section{Interpreting high-momentum scaling behavior of the momentum distribution}

As discussed in Sec. I, results from large-momentumtransfer experiments such as $\left(e, e^{\prime} p\right)$ have been related [15] to how the tails of momentum distributions calculated for nuclei and nuclear matter using phenomenological potentials exhibit a scaling behavior at high momentum [30]. In particular, the momentum dependence of the distributions for nuclei ranging from the deuteron to oxygen, as well as nuclear matter, at high 
momentum is similar except for an overall nucleus-dependent scaling factor. One explanation for this is based on the dominance of two-body forces in the interaction and shortrange correlations in the wave functions [15]. How can we explain this feature in an SRG-evolved calculation, for which high-momentum components and short-range correlations are suppressed?

Factorization provides a compelling alternative explanation. Because the $a_{q}^{\dagger} a_{q}$ operator for large $q$ has no coupling to low momentum, the entire $q$ dependence comes through the function $I_{Q O Q}$ in Eqs. (56) and (57), which is independent of the low-momentum part. If induced many-body contributions to the operator are relatively small and we neglect for the moment effects from embedding two-body operators into an $A>2$ space, we conclude that for $A \geqslant 2$ the momentum distribution should be approximately the same for every $A$, with a scaling factor given by an $A$-dependent low-momentum integral over the low-energy wave functions.

We have tested this proposal in a 1D model with an interaction that mimics features of the nuclear $N N$ potential. The model and procedures used for the 1D calculations are described in Ref. [12]. The full momentum distributions for two-, three-, and four-particle systems in this model are shown with solid, dot-dashed, and dashed lines, respectively, in Fig. 19. The behavior at high momentum is analogous to the nuclear calculations [30]: The momentum dependence is similar for each system so that each curve differs only by a scaling factor. We then evolve the model interaction via the SRG to $\lambda=2$ and extract the unitary transformation. Only the operator from $A=2$ is used; that is, any induced three- or four-body component is neglected. Details of the extraction and embedding of operators for $A>2$, including boost corrections, are described in the sequel [10]. By using the first term in the SVD expansion to factorize $U(k, q)$ in the

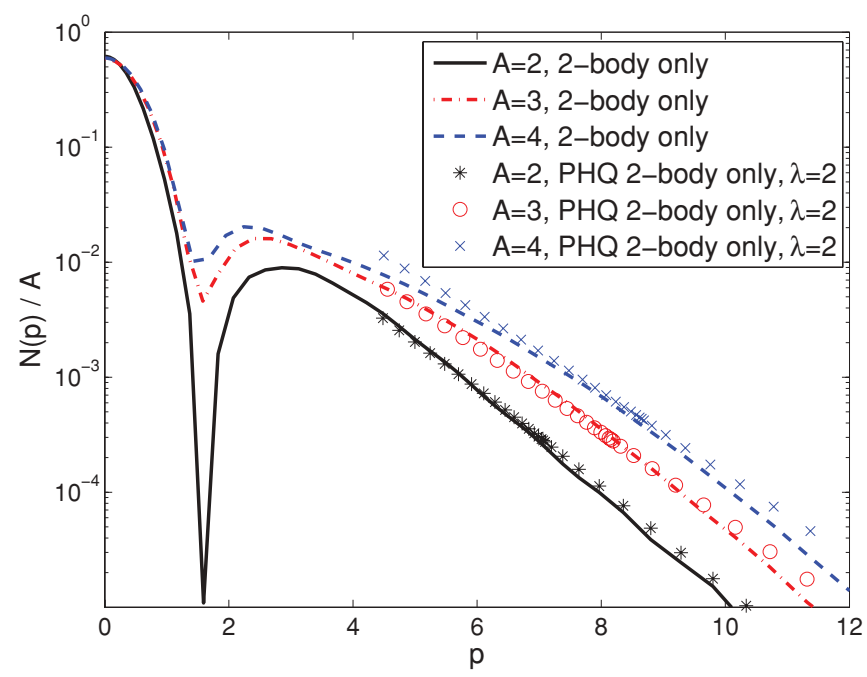

FIG. 19. (Color online) The scaling of momentum distributions at high momenta in a 1D model is tested by using the leading-order factorized approximation to the momentum occupation number operator to predict high-momentum scaling in $A=2,3,4$ (symbols). The full momentum distributions for $A=2,3$, and 4 are shown with solid, dot-dashed, and dashed lines, respectively. region where $k\langle\lambda$ and $q \gg \lambda$, we are able to reproduce to a large extent the momentum distributions at high momenta (shown with symbols in Fig. 19) and confirm our expectations regarding the scaling of the curves. These results are very promising and merit further investigation.

\section{SUMMARY}

In this article, we have examined the evolution of operators via the SRG with restricted application to the deuteron. We considered only the most commonly used generator $G_{s}=$ $\left[T_{\mathrm{rel}}, H_{s}\right]$ with normal ordering in the vacuum. At this twoparticle level it is easy to ensure that the transformations are unitary to high accuracy, so the invariance of matrix elements is assured. Thus, our focus is instead on the nature of the evolved operators: Does a form of decoupling apply? Do operators become increasingly complicated as the wave functions become increasingly less correlated? How large are induced two-body contributions to various one-body operators?

By considering the operator matrix elements in momentum representation both with and without deuteron wave functions included, we are able to follow the flow of strength. Because the transformations are unitary, the integrated value does not change with $\lambda$, but the nature of the operator does. There is little evolution in long-distance operators, whereas high-momentum operators must evolve significantly to compensate for suppression of high momenta in low-energy wave functions. In the end, one can see that the movement of the strength in the operator expectation values is given by the evolution of the eigenstates of the Hamiltonian itself. Moreover, we find that decoupling succeeds for operator expectation values in general, not just for the binding energies.

The momentum distribution is particularly interesting because the evolution of high-momentum operators leads to their strength flowing completely to low momentum. Thus while the deuteron wave function has rapidly decreasing support at the high momentum, its matrix elements are preserved without pathologies in the transformed operators. Indeed, operator matrix elements are less sensitive to details, as evidenced by the improved effectiveness of estimates using variational wave functions. Decoupling for operators follows as the contributions from higher energy/momentum basis states become unimportant, allowing truncation. This was explicitly illustrated in Figs. 5 and 6 . The generality of these conclusions is evident by considering the eigenvector expansion of the SRG unitary transformations, which dictates the flow of strength.

For low-momentum operators, which also includes the lowmomentum part of one-body electromagnetic form factors, there is relatively little running and therefore only small induced two-body parts (which for $A=2$ is simply the difference between the initial and the evolved result). In general, if the initial operator matrix elements pick up their strength predominantly at long distance, the operators will evolve only slightly until $\lambda$ is small. For electroweak operators, the real interest is in few- and many-body systems, where the simpler SRG-evolved wave functions are most advantageous.

The practical pathway to few-body operators and applications to $A>2$ is at present through the (Jacobi) 
harmonic-oscillator basis. The successful SRG evolution of three-body forces (and higher in model systems) in this basis is detailed in Refs. [8,12]. The corresponding challenge is to evolve operators for $A=2$ and $A=3$ and then embed them (including induced contributions) in higher- $A$ spaces. Procedures for carrying this out, including the need to boost some operators, will be discussed in the sequel to this article [10]. The calculation of transition matrix elements rather than ground-state expectation values requires separate consideration.

Of particular interest will be the further study of SRG operator factorization, which occurs when there is a scale separation between the initial (unevolved) operator and the wave-function momentum scale, which is limited by $\lambda$. This factorization was shown to be a natural consequence of applying the OPE to the unitary transformation. The extension to $A>2$ was previewed in an application of factorization for momentum distributions of low-energy bound states, which provided an alternative interpretation to the commonly invoked role of short-range correlations. Work is in progress on the full realistic three-dimensional calculations.

In closing, we reiterate that the favorable consequences of the SRG is a specific realization of the more general observation that the RG allows one to focus on the most relevant degrees of freedom in a physical problem [31]. Thus, an evolution to low momentum for nuclear systems can be win-win not only for the Hamiltonian and wave functions but for operators as well. The SRG has some special advantages in practice because it uses operator flow equations that can be applied in any convenient basis with a variety of options for tailoring the flow. Furthermore, residual dependence on the flow parameter $s$ or $\lambda$ becomes a powerful tool for assessing approximations. Different resolutions can lead to very different physical interpretations and intuition; the RG has the great advantage of being able to connect the different pictures.

\section{ACKNOWLEDGMENTS}

We thank E. Jurgenson, A. Schwenk, and K. Wendt for useful comments and discussions. We also thank E. Jurgenson for the use of his 1D few-body code. This work was supported in part by the National Science Foundation under Grants No. PHY-0653312 and No. PHY-0758125 and the UNEDF SciDAC Collaboration under DOE Grants No. DE-FC0209ER41457 and No. DE-FC02-09ER41585.
[1] S. K. Bogner, R. J. Furnstahl, and A. Schwenk, Prog. Part. Nucl. Phys. 65, 94 (2010).

[2] S. D. Glazek and K. G. Wilson, Phys. Rev. D 48, 5863 (1993).

[3] F. Wegner, Ann. Phys. (Leipzig) 506, 77 (1994).

[4] S. Szpigel and R. J. Perry, in Quantum Field Theory: A 20th Century Profile, edited by A. N. Mitra (Hindustan Publishing Co., New Delhi, 2000), pp. 59-81.

[5] S. D. Glazek and T. Maslowski, Phys. Rev. D 65, 065011 (2002).

[6] S. K. Bogner, R. J. Furnstahl, and R. J. Perry, Phys. Rev. C 75, 061001 (2007).

[7] E. D. Jurgenson, S. K. Bogner, R. J. Furnstahl, and R. J. Perry, Phys. Rev. C 78, 014003 (2008).

[8] E. D. Jurgenson, P. Navratil, and R. J. Furnstahl, Phys. Rev. Lett. 103, 082501 (2009).

[9] E. Anderson et al., Phys. Rev. C 77, 037001 (2008).

[10] E. Anderson, E. J. Jurgenson, R. J. Furnstahl, and R. J. Perry (in preparation).

[11] S. K. Bogner et al., Nucl. Phys. A 801, 21 (2008).

[12] E. D. Jurgenson and R. J. Furnstahl, Nucl. Phys. A 818, 152 (2009).

[13] S. K. Bogner, R. J. Furnstahl, R. J. Perry, and A. Schwenk, Phys. Lett. B 649, 488 (2007).

[14] K. Tsukiyama, S. K. Bogner, and A. Schwenk, arXiv:1006.3639 [nucl-th].

[15] L. Frankfurt, M. Sargsian, and M. Strikman, Int. J. Mod. Phys. A 23, 2991 (2008).
[16] R. B. Wiringa, V. G. J. Stoks, and R. Schiavilla, Phys. Rev. C 51, 38 (1995).

[17] D. R. Entem and R. Machleidt, Phys. Rev. C 68, 041001 (2003).

[18] S. K. Bogner, R. J. Furnstahl, S. Ramanan, and A. Schwenk, Nucl. Phys. A 784, 79 (2007).

[19] I. Sick, Prog. Part. Nucl. Phys. 47, 245 (2001).

[20] M. Garcon and J. W. Van Orden, Adv. Nucl. Phys. 26, 293 (2001).

[21] R. A. Gilman and F. Gross, J. Phys. G 28, R37 (2002).

[22] D. R. Phillips, J. Phys. G 34, 365 (2007).

[23] M. P. Valderrama, A. Nogga, E. Ruiz Arriola, and D. R. Phillips, Eur. Phys. J. A 36, 315 (2008).

[24] M. A. Belushkin, H. W. Hammer, and U. G. Meissner, Phys. Rev. C 75, 035202 (2007).

[25] E. Epelbaum, W. Glockle, and U.-G. Meissner, Nucl. Phys. A 747, 362 (2005).

[26] S. K. Bogner and R. J. Furnstahl, Phys. Lett. B 632, 501 (2006).

[27] S. K. Bogner and R. J. Furnstahl, Phys. Lett. B 639, 237 (2006).

[28] P. Lepage, arXiv:nucl-th/9706029v1.

[29] E. Braaten and L. Platter, Phys. Rev. Lett. 100, 205301 (2008).

[30] S. C. Pieper, R. B. Wiringa, and V. R. Pandharipande, Phys. Rev. C 46, 1741 (1992).

[31] Asymptotic Realms of Physics, Essays in Honor of Francis E. Low, edited by Alan H. Guth, Kerson Huang, and Robert L. Jaffe (The MIT Press, Cambridge, MA, 1983), p. 1. 